\title{
Well-posed conditions on a class of fractional $q$-differential equations by using the Schauder fixed point theorem
}

\author{
Mohammad Esmael Samei ${ }^{1}$, Ahmad Ahmadi ${ }^{1}$, A. George Maria Selvam², Jehad Alzabut ${ }^{3,4^{*}}$ and \\ Shahram Rezapour ${ }^{5,6^{*}}$ (I)
}

"Correspondence:

jalzabut@psu.edu.sa;

sh.rezapour@azaruniv.ac.ir;

sh.rezapour@mail.cmuh.org.tw; rezapourshahram@yahoo.ca

3epartment of Mathematics and

General Sciences, Prince Sultan

University, Riyadh, Saudi Arabia

${ }^{5}$ Department of Mathematics,

Azarbaijan Shahid Madani

University, Tabriz, Iran

Full list of author information is

available at the end of the article

\section{Springer}

\begin{abstract}
In this paper, we propose the conditions on which a class of boundary value problems, presented by fractional $q$-differential equations, is well-posed. First, under the suitable conditions, we will prove the existence and uniqueness of solution by means of the Schauder fixed point theorem. Then, the stability of solution will be discussed under the perturbations of boundary condition, a function existing in the problem, and the fractional order derivative. Examples involving algorithms and illustrated graphs are presented to demonstrate the validity of our theoretical findings.
\end{abstract}

MSC: $34 \mathrm{~A} 08 ; 34 \mathrm{~B} 16 ; 34 \mathrm{~B} 18$

Keywords: Fractional q-derivative equations; Nonlinear analysis theorems; Well-posedness

\section{Introduction}

In many applications fractional differential equations present more accurate models of phenomena than the ordinary differential equations. Therefore they have obtained importance due to their applications in science and engineering such as, physics, chemistry, mechanics, fluid dynamic, etc. [1, 2]. Meanwhile, there have appeared many papers dealing with the existence of solutions for different types of fractional boundary value problems; see, for example, [3-19].

The quantum calculus was introduced by Jackson in 1910 [20]. Al-Salam started the fitting of the concept of $q$-fractional calculus [21]. Then Agarwal continued by studying certain $q$-fractional integrals and derivatives [22]. After it, some researchers studied $q$ difference equations (for more details, see [23-36]). There are also many papers dealing with the existence of solutions for $q$-fractional boundary value problems (see, for example, [37-49]).

Existence of solutions to fractional differential equations has received considerable interest in recent years. There are several papers dealing with the existence and uniqueness of solutions to initial and boundary value problem of fractional order in Caputo or

(c) The Author(s) 2021. This article is licensed under a Creative Commons Attribution 4.0 International License, which permits use sharing, adaptation, distribution and reproduction in any medium or format, as long as you give appropriate credit to the original author(s) and the source, provide a link to the Creative Commons licence, and indicate if changes were made. The images or other third party material in this article are included in the article's Creative Commons licence, unless indicated otherwise in a credit line to the material. If material is not included in the article's Creative Commons licence and your intended use is not permitted by statutory regulation or exceeds the permitted use, you will need to obtain permission directly from the copyright holder. To view a copy of this licence, visit http://creativecommons.org/licenses/by/4.0/ 
Riemann-Liouville sense (for more details, see [50-52] and the references therein). Some authors have also investigated the existence and uniqueness solutions for a coupled system of multi-term fractional differential equations $[53,54]$. However, in general, the study of well-posed conditions for fractional differential equations is less considered in the literature.

In 2015, Houas et al. [55] investigated the existence and uniqueness of solutions for ${ }^{c} \mathcal{D}_{q}^{\sigma}[y](t)+w\left(y(t),{ }^{c} \mathcal{D}_{q}^{\varsigma}[y](t)\right)=0$, for $t \in \bar{J}_{0}:=[0,1]$, where $2<\sigma \leq 3, \varsigma \in J_{0}:=(0,1)$, under the initial conditions $y(0)=y_{0}, y^{\prime}(0)=0, y^{\prime}(1)=\eta \mathcal{I}^{\zeta} y(e)$, where ${ }^{c} \mathcal{D}^{\sigma}$ is the Caputo fractional derivative, $e \in J_{0}, w$ is a continuous function on $\mathbb{R}^{2}$, and $\eta$ is a real constant [55]. In [56], authors studied the existence and uniqueness of solution for the fractional differential equation $\mathcal{D}^{\sigma}[y](t)=w\left(t, y(t), \mathcal{D}^{\varsigma}[y](t)\right)$, where $2<\sigma<3, \varsigma \in J_{0}$, via sum boundary conditions $y(0)=0$,

$$
\mathcal{D}_{q}^{\sigma}[y](1)=\sum_{i=1}^{m-2} a_{i} \mathcal{D}_{q}^{\varsigma}[y]\left(e_{i}\right),
$$

$y^{\prime \prime}(1)=0$, where, $a_{i}, e_{i} \in J_{0}$ and $\mathcal{D}_{q}^{\sigma}$ is the Caputo fractional derivative. In 2015, Akrami et al. [57] proved the conditions on which the following class of fractional differential equations ${ }^{C} \mathcal{D}^{\sigma}[y](t)=w\left(y(t), \mathcal{D}^{\varsigma}[y](t)\right)$ for $t \in \bar{J}_{0}$ is well-posed, where $2<\sigma \leq 3$ and $\varsigma \in J_{0}$, and ${ }^{C} \mathcal{D}_{q}^{\sigma}$ is the Caputo fractional derivative subject to the boundary value conditions $y(0)=y^{\prime}(0)=0$, $y(1)=a y(e)$, where $e \in J_{0}, 0 \leq a<\frac{1}{e^{2}}$.

In this article, we investigate the conditions on which the fractional $q$-differential equation

$$
{ }^{C} \mathcal{D}_{q}^{\sigma}[y](t)=w\left(y(t),{ }^{C} \mathcal{D}_{q}^{\varsigma}[y](t)\right)
$$

for $t \in \bar{J}_{0}$ is well-posed, where $2<\sigma \leq 3, \varsigma \in J_{0}$, and ${ }^{C} \mathcal{D}_{q}^{\sigma}$ is the standard Caputo $q$ derivative subject to the boundary value conditions

$$
y(0)=y^{\prime}(0)=0, \quad y^{\prime}(1)=a y(e),
$$

where $e \in \bar{J}_{0}$ with $0 \leq a<\frac{1}{e^{2}}$. We recall that a problem is said to be well-posed if it has a uniqueness solution and this solution depends on a parameter in a continuous way. This parameter, in the classical order differential equations, is dependent on the initial conditions and the function exists in the problem; whereas in the FDEs this dependency and the stability solution with respect to the perturbation of fractional order derivative should be taken into the account too [58].

The rest of the paper is organized as follows. We first prove the existence solution of (1) by means of the Schauder fixed point theorem on the interval $\bar{J}_{0}$ in Sect. 3 . Then, we prove the uniqueness by using the Banach contraction map theorem under a suitable condition in Sect. 3. Also, Sect. 3 is devoted to investigating the stability of solutions under the perturbations on boundary condition, the function exists in the problem and the fractional order derivative. Finally, in Sect. 4, we bring some examples to illustrate our results. Let us start with some basic preliminaries in Sect. 2 that we will use in the sequel. 


\section{Preliminaries and lemmas}

This section is devoted to some notations and essential preliminaries that are acting as necessary prerequisites for the results of the subsequent sections. Throughout the context, we shall apply the notations of time scales calculus [59].

In fact, we consider the fractional $q$-calculus on the specific time scale $\mathbb{T}_{t_{0}}=\{0\} \cup\{t$ : $\left.t=t_{0} q^{n}\right\}$ for $n \in \mathbb{N}, t_{0} \in \mathbb{R}$, and $q \in(0,1)$. For brief, we shall denote $\mathbb{T}_{t_{0}}$ by $\mathbb{T}$. Let $a \in \mathbb{R}$. Define $[s]_{q}=\left(1-q^{s}\right) /(1-q)$ [20]. The $q$-factorial function $(v-w)_{q}^{(n)}$ with $n \in \mathbb{N}_{0}:=\{0\} \cup \mathbb{N}$ is defined by $(v-w)_{q}^{(n)}=\prod_{k=0}^{n-1}\left(v-w q^{k}\right)$, and $(v-w)_{q}^{(0)}=1$, where $v, w$ are real numbers [23]. Also, for $\sigma \in \mathbb{R}$ and $s \neq 0$, we have $(v-w)_{q}^{(\sigma)}=v^{\sigma} \prod_{k=0}^{\infty}\left(v-w q^{k}\right)\left(v-w q^{-(\sigma+k)}\right)$. In the paper [60], the authors proved $(v-w)_{q}^{(\sigma+v)}=(v-w)_{q}^{(\sigma)}\left(v-q^{\sigma} w\right)_{q}^{(\nu)}$ and $(s v-s w)_{q}^{(\sigma)}=$ $s^{\sigma}(v-w)_{q}^{(\sigma)}$. If $w=0$, then it is clear that $v^{(\sigma)}=v^{\sigma}$. The $q$-gamma function is given by $\Gamma_{q}(v)=(1-q)^{1-v}(1-q)_{q}^{(v-1)}$, where $z \in \mathbb{R} \backslash\{\ldots,-2,-1,0\}[20]$. Note that $\Gamma_{q}(z+1)=[z]_{q} \Gamma_{q}(z)$ $[60$, Lemma 1$]$. For a function $\wp: \mathbb{T} \rightarrow \mathbb{R}$, the $q$-derivative of $\wp$ is

$$
\mathcal{D}_{q}[\wp](v)=\frac{\wp(v)-\wp(q v)}{(1-q) v}
$$

for all $t \in \mathbb{T} \backslash\{0\}$, and $\mathcal{D}_{q}[\wp](0)=\lim _{v \rightarrow 0} \mathcal{D}_{q} \wp(v)$ [23]. Also, the higher order $q$-derivative of the function $\wp$ is defined by $\mathcal{D}_{q}^{n} \wp(v)=\mathcal{D}_{q}\left(\mathcal{D}_{q}^{n-1} \wp\right)(v)$ for all $n \geq 1$, where $\mathcal{D}_{q}^{0} \wp(v)=\wp(v)$ [23]. The $q$-integral of the function $\wp$ is defined by

$$
\mathcal{I}_{q} \wp(v)=\int_{0}^{v} \wp(\xi) \mathrm{d}_{q} \xi=v(1-q) \sum_{k=0}^{\infty} q^{k} \wp\left(v q^{k}\right)
$$

for $0 \leq v \leq b$, provided the series absolutely converges [23]. If $v$ in $[0, b]$, then

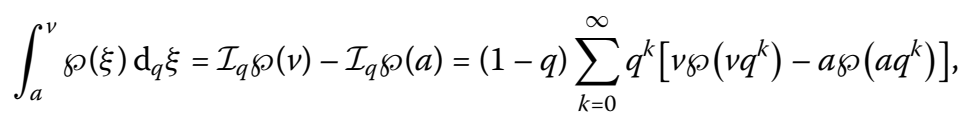

whenever the series exists [61]. The operator $\mathcal{I}_{q}^{n}$ is given by $\mathcal{I}_{q}^{0} \wp(v)=\wp(v)$ and $\mathcal{I}_{q}^{n} \wp(v)=$ $\mathcal{I}_{q}\left[\mathcal{I}_{q}^{n-1} \wp\right](v)$ for $n \geq 1$ and $\wp \in C([0, b])$ [23]. It has been proved that $\mathcal{D}_{q}\left(\mathcal{I}_{q} \wp\right)(v)=\wp(v)$, $\mathcal{I}_{q}\left(\mathcal{D}_{q} \wp\right)(v)=\wp(v)-\wp(0)$, whenever the function $\wp$ is continuous at $v=0$ [23]. The fractional Riemann-Liouville type $q$-integral of the function $\wp$ is defined by $\mathcal{I}_{q}^{0} \wp(v)=\wp(v)$ and

$$
\mathcal{I}_{q}^{\sigma} \wp(v)=\frac{1}{\Gamma_{q}(\sigma)} \int_{0}^{v}(v-\xi)_{q}^{(\sigma-1)} \wp(\xi) \mathrm{d}_{q} \xi
$$

for $v \in[0,1]$ and $\sigma>0[24,29]$. The Caputo fractional $q$-derivative of the function $\wp$ is defined by

$$
{ }^{C} \mathcal{D}_{q}^{\sigma} \wp(v)=\mathcal{I}_{q}^{[\sigma]-\sigma}\left[\mathcal{D}_{q}^{[\sigma]} \wp\right](v)=\frac{1}{\Gamma_{q}([\sigma]-\sigma)} \int_{0}^{v}(\nu-\xi)_{q}^{([\sigma]-\sigma-1)} \mathcal{D}_{q}^{[\sigma]} \wp(\xi) \mathrm{d}_{q} \xi
$$

for $v \in[0,1]$ and $\sigma>0[29,62]$. It has been proved that $\mathcal{I}_{q}^{v}\left(\mathcal{I}_{q}^{\sigma} \wp\right)(v)=\mathcal{I}_{q}^{\sigma+v} \wp(v)$ and ${ }^{c} \mathcal{D}_{q}^{\sigma}\left(\mathcal{I}_{q}^{\sigma} \wp\right)(v)=\wp(v)$, where $\sigma, v \geq 0$ [29]. The authors in [61] presented all algorithms and MATLAB lines to simplify $q$-factorial functions $(v-w)_{q}^{(n)},(v-w)_{q}^{(\sigma)}, \Gamma_{q}(v), \mathcal{I}_{q}[\wp](v)$, and some necessary equations. 
Now, we introduce some basic definitions, lemmas, and theorems which are used in the subsequent sections.

Lemma 2.1 ([63]) Let $n \in \mathbb{N}, n-1<\alpha \leq n$, and $\wp \in A C^{n}[a, b]$. Then one has $\mathcal{I}^{\sigma}\left({ }^{c} \mathcal{D}_{q}^{\sigma} \wp\right)(v)=$ $\wp(v)+\sum_{i=0}^{n-1} c_{i}(v-a)^{i}$, where $c_{0}, c_{1}, \ldots, c_{n-1} \in \mathbb{R}$.

Lemma 2.2 Let $\sigma_{1}>\sigma_{2}>0$. Then the formula ${ }^{C} \mathcal{D}_{q}^{\sigma_{1}}\left(\mathcal{I}_{q}^{\sigma_{2}} \wp\right)(v)=\mathcal{I}_{q}^{\sigma_{1}-\sigma_{2}} \wp(v)$ holds almost everywhere on $v \in[a, b]$ for $\wp \in L_{1}[a, b]$, and it is valid at any point $v \in[a, b]$ if $\wp \in C([a, b], \mathbb{R})$.

Lemma 2.3 ([1]) Let $\sigma>0$ and $\wp \in C(0,1) \cap L^{1}(0,1)$ with a derivative of order $n$. Then $\mathcal{I}_{q}^{\sigma}\left({ }^{C} \mathcal{D}_{q}^{\sigma} \wp(v)\right)=\wp(v)+d_{0}+d_{1} t+d_{2} t^{2}+\cdots+d_{n-1} t^{n-1}$ for $d_{i} \in \mathbb{R}$ with $i=1,2, \ldots, n-1$, where $n-1<\sigma \leq n$.

Definition 2.4 A real function $\wp(v), v>0$ is said to be in the space $C_{r}, r \in \mathbb{R}$, if there exists a real number $v(>r)$ such that $\wp(v)=v^{v} \wp_{1}(v)$, where $\wp_{1}(v) \in C([0, \infty), \infty)$.

Theorem 2.5 (Banach contraction principle, [64]) Let $\mathcal{X}$ be a Banach space. If $A: \mathcal{X} \rightarrow \mathcal{X}$ is the contraction map, then there exists $x \in \mathcal{X}$ such that $A x=x$.

\section{Main results}

First, we consider the following important lemmas in our article.

Lemma 3.1 Let $v \in A C(0,1)$ and $2<\sigma \leq 3$. The fractional q-differential equation

$$
{ }^{C} \mathcal{D}_{q}^{\sigma}[y](t)=v(t)
$$

for $2<\sigma \leq 3$ under conditions $y(0)=y^{\prime}(0)=0, y^{\prime}(1)=a y(e), e \in J_{0}$ with $0 \leq a<\frac{1}{e^{2}}$ has a solution

$$
y(t)=\int_{0}^{1} G_{q}(t, \xi) v(\xi) \mathrm{d}_{q} \xi+\frac{a t^{2}}{1-a e^{2}} \int_{0}^{1} G_{q}(e, \xi) v(\xi) \mathrm{d}_{q} \xi,
$$

where

$$
G_{q}(t, \xi)= \begin{cases}\frac{(t-\xi)_{q}^{(\sigma-1)}-t^{2}(1-\xi)_{q}^{(\alpha-1)}}{\Gamma_{q}(\sigma)}, & \xi<t, \\ \frac{-t^{2}(1-\xi)_{q}^{(\sigma-1)}}{\Gamma_{q}(\sigma)}, & t<\xi,\end{cases}
$$

for all $t, \xi \in \bar{J}_{0}$.

Proof By Lemma (2.3) the solution of Eq. (3) can be written as

$$
y(t)=\int_{0}^{t}(t-\xi)_{q}^{(\sigma-1)} v(\xi) \mathrm{d}_{q} \xi-d_{0}-d_{1} t-d_{2} t^{2} .
$$

Since $y(0)=y^{\prime}(0)=0$, a simple calculation gives $d_{0}-d_{1}=0$, and from the boundary condition, we get $\mathcal{I}_{q}^{\sigma}[v](1)-d_{2}=a \mathcal{I}_{q}^{\sigma}[v](e)-d_{2} a e^{2}$. Hence,

$$
d_{2}=\frac{1}{1-a e^{2}}\left(\mathcal{I}_{q}^{\sigma}[v](1)-a \mathcal{I}_{q}^{\sigma}[v](e)\right) .
$$


Thus, the solution of boundary value problem (3) is

$$
\begin{aligned}
y(t)= & \mathcal{I}_{q}^{\sigma}[v](t)-\frac{t^{2}}{1-a e^{2}}\left(\mathcal{I}_{q}^{\sigma}[v](1)-a \mathcal{I}_{q}^{\sigma}[v](e)\right) \\
= & \mathcal{I}_{q}^{\sigma}[v](t)-t^{2} \mathcal{I}_{q}^{\sigma}[v](1)-\frac{a e^{2} t^{2}}{1-a e^{2}} \mathcal{I}_{q}^{\sigma}[v](1)+\frac{a t^{2}}{1-a e^{2}} \mathcal{I}_{q}^{\sigma}[v](e) \\
= & \frac{1}{\Gamma_{q}(\sigma)} \int_{0}^{t}\left((t-\xi)_{q}^{(\sigma-1)}-t^{2}(1-\xi)_{q}^{(\sigma-1)}\right) v(\xi) \mathrm{d}_{q} \xi \\
& -\frac{1}{\Gamma_{q}(\sigma)} \int_{1}^{t} t^{2}(1-\xi)_{q}^{(\sigma-1)} v(\xi) \mathrm{d}_{q} \xi \\
& +\frac{a t^{2}}{\left(1-a e^{2}\right) \Gamma_{q}(\sigma)}\left[\int_{0}^{e}\left((e-\xi)_{q}^{(\sigma-1)}-e^{2}(1-\xi)_{q}^{(\sigma-1)}\right) v(\xi) \mathrm{d}_{q} \xi\right. \\
& \left.-\int_{e}^{t} e^{2}(1-\xi)_{q}^{(\sigma-1)} v(\xi) \mathrm{d}_{q} \xi\right] \\
= & \int_{0}^{1} G_{q}(t, \xi) v(\xi) \mathrm{d}_{q} \xi+\frac{a t^{2}}{1-a e^{2}} \int_{0}^{1} G_{q}(e, \xi) v(\xi) \mathrm{d}_{q} \xi,
\end{aligned}
$$

where $G_{q}(t, \xi)$ is defined in Eq. (5). This completes the proof.

Now, in order to investigate the existence of solutions, we prove some properties of the function $G_{q}(t, \xi)$.

Lemma 3.2 The functions $G_{q}(t, \cdot)$ and $\frac{\partial}{\partial t} G_{q}(t, \cdot)$ are integrable for each $t \in \bar{J}_{0}$ and have the following properties:

$$
\int_{0}^{1}\left|G_{q}(t, \xi)\right| \mathrm{d}_{q} \xi \leq \frac{2}{\Gamma_{q}(\sigma+1)}, \int_{0}^{1}\left|\frac{\partial}{\partial t} G_{q}(t, \xi)\right| \mathrm{d}_{q} \xi \leq \frac{3}{\Gamma_{q}(\sigma)}
$$

Proof Let $t \in \bar{J}_{0}$. Then we have

$$
\begin{aligned}
\int_{0}^{1}\left|G_{q}(t, \xi)\right| \mathrm{d}_{q} \xi & \leq \mathcal{I}_{q}^{\sigma}[I](t)++t^{2} \mathcal{I}_{q}^{\sigma}[I](1) \\
& \leq \frac{t^{\sigma}}{\Gamma_{q}(\sigma+1)}+\frac{t^{2}}{\Gamma_{q}(\sigma+1)} \leq \frac{2}{\Gamma_{q}(\sigma+1)}
\end{aligned}
$$

and

$$
\begin{aligned}
\int_{0}^{1}\left|\frac{\partial}{\partial t} G_{q}(t, \xi)\right| \mathrm{d}_{q} \xi & \leq 2 t \mathcal{I}_{q}^{\sigma}[I](1)++\mathcal{I}_{q}^{\sigma-1}[I](t) \\
& \leq \frac{2 t}{\Gamma_{q}(\sigma+1)}+\frac{t^{\sigma-1}}{\Gamma_{q}(\sigma)} \leq \frac{3}{\Gamma_{q}(\sigma)}
\end{aligned}
$$

Hence, $G_{q}(t, \cdot)$ and $\frac{\partial}{\partial t} G_{q}(t, \cdot)$ are integrable.

Let $C^{1}\left(\bar{J}_{0}\right)$ be the class of all continuous functions. Since ${ }^{C} \mathcal{D}_{q}^{\varsigma}[y](t)=\mathcal{I}_{q}^{1-\varsigma}\left[y^{\prime}\right](t)$ for $\varsigma \in$ $J_{0}$, the operator ${ }^{C} \mathcal{D}_{q}^{S}$ is continuous for any $y \in C^{1}\left(\bar{J}_{0}\right)$. Now, for $y \in C^{1}\left(\bar{J}_{0}\right)$, we define the 
space

$$
\mathcal{A}=\left\{y(t): y(t) \in C^{1}\left(\bar{J}_{0}\right)\right\}
$$

endowed with the maximum norm

$$
\|y\|=\max _{t \in \bar{J}_{0}}|y(t)|+\max _{t \in \bar{J}_{0}}\left|{ }^{C} \mathcal{D}_{q}^{s}[y](t)\right| .
$$

Lemma 3.3 $(\mathcal{A},\|\cdot\|)$ is a Banach space.

Proof Let $\left\{y_{n}\right\}_{n=1}^{\infty}$ be a Cauchy sequence in the space $(\mathcal{A},\|\cdot\|)$. Obviously, $\left\{y_{n}\right\}_{n=1}^{\infty}$ and $\left\{{ }^{C} \mathcal{D}_{q}^{S} y_{n}\right\}_{n=1}^{\infty}$ are Cauchy sequences in the space $C\left(\bar{J}_{0}\right)$. Since $C\left(\bar{J}_{0}\right)$ is compact, $\left\{y_{n}\right\}_{n=1}^{\infty}$ and $\left\{{ }^{C} \mathcal{D}_{q}^{S} y_{n}\right\}_{n=1}^{\infty}$ uniformly converge to some $v, v^{\prime}$ on $\bar{J}_{0}$. Furthermore, $v, v^{\prime}$ belong to $C\left(\bar{J}_{0}\right)$. In the following, we need to show that $v^{\prime}={ }^{C} \mathcal{D}_{q}^{S} v$. Now, by the definition of fractional integral,

$$
\begin{aligned}
\left|\mathcal{I}_{q}^{\varsigma}\left[{ }^{C} \mathcal{D}_{q}^{\varsigma}\left[y_{n}\right]\right](t)-\mathcal{I}_{q}^{\varsigma} v^{\prime}(t)\right| & \leq \mathcal{I}_{q}^{\varsigma}\left[\left|{ }^{C} \mathcal{D}_{q}^{\varsigma} y_{n}-v^{\prime}\right|\right](t) \\
& \leq\left.\frac{1}{\Gamma_{q}(\varsigma+1)} \max _{t \in \bar{J}_{0}}\right|^{C} \mathcal{D}_{q}^{\varsigma} y_{n}-v^{\prime} \mid
\end{aligned}
$$

Therefore, using the convergence of $\left\{{ }^{C} \mathcal{D}_{q}^{S} y_{n}\right\}_{n=1}^{\infty}$ implies that

$$
\lim _{n \rightarrow \infty} \mathcal{I}_{q}^{\varsigma}\left[{ }^{C} \mathcal{D}_{q}^{\varsigma}\left[y_{n}\right]\right](t)=\mathcal{I}_{q}^{\varsigma}\left[v^{\prime}\right](t)
$$

uniformly on $\bar{J}_{0}$. On the other hand, we know $\mathcal{I}_{q}^{\varsigma}\left[{ }^{C} \mathcal{D}_{q}^{\varsigma}\left[y_{n}\right]\right](t)=y_{n}$ for each $t \in \bar{J}_{0}$ and $\varsigma \in J_{0}$. Hence, $\mathcal{I}_{q}^{S}\left[v^{\prime}\right](t)=v$, and this means $v^{\prime}={ }^{C} \mathcal{D}_{q}^{S} v$. This completes the proof.

Remark 3.1 Lemma (2.3) implies that the solution of problem (1) coincides with the fixed point of the operator $\mathcal{O}$ defined as

$$
\begin{aligned}
\mathcal{O} y(t)= & \int_{0}^{1} G_{q}(t, \xi) w\left(y(t),{ }^{C} \mathcal{D}_{q}^{\varsigma}[y](t)\right) \mathrm{d}_{q} \xi \\
& +\frac{a t^{2}}{1-a e^{2}} \int_{0}^{1} G_{q}(e, \xi) w\left(y(t),{ }^{C} \mathcal{D}_{q}^{S}[y](t)\right) \mathrm{d}_{q} \xi .
\end{aligned}
$$

\subsection{Existence and uniqueness}

According to the Schauder fixed point theorem, the existence result has been stated.

Theorem 3.4 Suppose that $w: \mathbb{R}^{2} \rightarrow \mathbb{R}$ is a continuous function and there exist constants $m_{0}, m_{1} \geq 0, \beta_{0}, \beta_{1} \in J_{0}$ such that one of the following conditions is satisfied:

(A1) There exists a nonnegative function $\mu(t) \in \bar{J}_{0}$ such that

$$
|w(y, z)| \leq \mu(t)+m_{0}|y|^{\beta_{0}}+m_{1}|z|^{\beta_{1}} .
$$

(A2) The function $w$ satisfies

$$
|w(y, z)| \leq m_{0}|y|^{\beta_{0}}+m_{1}|z|^{\beta_{1}}
$$

Then boundary value problem (1) has at least one solution $y(t)$. 
Proof First, suppose that condition (A1) holds. Define the set $\mathcal{B}$ by

$$
\mathcal{B}=\left\{y(t):\|y(t)\| \leq \delta, t \in \bar{J}_{0}\right\}
$$

where

$$
\begin{aligned}
\delta \geq & \max \left\{\left(6 \Delta m_{0}\right)^{\frac{1}{1-\beta_{0}}},\left(6 \Delta m_{1}\right)^{\frac{1}{1-\beta_{1}}}, 6 \Delta M_{1},\left(\frac{12 \Delta m_{0}}{\Gamma_{q}(2-\varsigma)}\right)^{\frac{1}{1-\beta_{0}}},\right. \\
& \left.\left(\frac{12 \Delta m_{1}}{\Gamma_{q}(2-\varsigma)}\right)^{\frac{1}{1-\beta_{1}}}, \frac{16 a M_{1}}{\Gamma_{q}(2-\varsigma)\left(1-a e^{2}\right)}, \frac{8 M_{2}}{\Gamma_{q}(2-\varsigma)}\right\}, \\
\Delta= & \left(1+\frac{a}{1-a e^{2}}\right) \frac{2}{\Gamma_{q}(\sigma+1)},
\end{aligned}
$$

and

$$
\begin{aligned}
& M_{1}=\max _{t \in \bar{J}_{0}}\left\{\frac{1}{\Gamma_{q}(\sigma)} \int_{0}^{1}\left|G_{q}(t, \xi) \mu(\xi)\right| \mathrm{d}_{q} \xi\right\} \\
& M_{2}=\max _{t \in \bar{J}_{0}}\left\{\frac{1}{\Gamma_{q}(\sigma)} \int_{0}^{1}\left|\frac{\partial}{\partial t} G_{q}(t, \xi) \mu(\xi)\right| \mathrm{d}_{q} \xi\right\} .
\end{aligned}
$$

It is clear that $\mathcal{B}$ is a closed, bounded, and convex subset of Banach space $\mathcal{A}$. Here, we prove that $\mathcal{O}: \mathcal{B} \rightarrow \mathcal{B}$. For any $y \in \mathcal{B}$, we obtain

$$
\begin{aligned}
|\mathcal{O} y(t)| \leq & \int_{0}^{1}\left|G_{q}(t, \xi) w\left(y(t),{ }^{C} \mathcal{D}_{q}^{\zeta}[y](t)\right)\right| \mathrm{d}_{q} \xi \\
& +\frac{a t^{2}}{1-a e^{2}} \int_{0}^{1}\left|G_{q}(e, \xi) w\left(y(t),{ }^{C} \mathcal{D}_{q}^{s}[y](t)\right)\right| \mathrm{d}_{q} \xi \\
\leq & \int_{0}^{1}\left|G_{q}(t, \xi) \mu(\xi)\right| \mathrm{d}_{q} \xi+\left[m_{0} \delta^{\beta_{0}}+m_{1} \delta^{\beta_{1}}\right] \int_{0}^{1}\left|G_{q}(t, \xi)\right| \mathrm{d}_{q} \xi \\
& +\frac{a}{1-a e^{2}}\left[\int_{0}^{1}\left|G_{q}(e, \xi) \mu(\xi)\right| \mathrm{d}_{q} \xi\right. \\
& \left.+\left(m_{0} \delta^{\beta_{0}}+m_{1} \delta^{\beta_{1}}\right) \int_{0}^{1}\left|G_{q}(t, \xi)\right| \mathrm{d}_{q} \xi\right] \\
\leq & \left(1+\frac{a}{1-a e^{2}}\right)\left[M_{1}+\frac{2}{\Gamma_{q}(\sigma+1)}\left(m_{0} \delta^{\beta_{0}}+m_{1} \delta^{\beta_{1}}\right)\right] \\
\leq & \Delta\left[M_{1}+\left(m_{0} \delta^{\beta_{0}}+m_{1} \delta^{\beta_{1}}\right)\right] \leq \frac{1}{2} \delta .
\end{aligned}
$$

Thus, for almost all $\varsigma \in J_{0}$, we have

$$
\begin{aligned}
\left|{ }^{C} \mathcal{D}_{q}^{\varsigma}[\mathcal{O} y](t)\right|= & \left|\mathcal{I}_{q}^{1-\varsigma}\left[\mathcal{O} y^{\prime}\right](t)\right| \\
\leq & \frac{1}{\Gamma_{q}(1-\varsigma)} \int_{0}^{t}(t-\xi)_{q}^{(-\varsigma)} \\
& \times\left(\int_{0}^{1}\left|\frac{\partial}{\partial \xi} G_{q}(\xi, \tau) w\left(\tau, y(\tau),{ }^{C} \mathcal{D}_{q}^{\varsigma}[y](\tau)\right)\right| \mathrm{d}_{q} \tau\right.
\end{aligned}
$$




$$
\begin{aligned}
& \left.+\frac{2 a \xi}{\left(1-a e^{2}\right)} \int_{0}^{1}\left|G_{q}(e, \tau) w\left(\tau, y(\tau),{ }^{C} \mathcal{D}_{q}^{\varsigma}[y](\tau)\right)\right| \mathrm{d}_{q} \tau\right) \mathrm{d}_{q} \xi \\
& \leq \frac{1}{\Gamma_{q}(1-\varsigma)} \int_{0}^{t}(t-q \xi)^{(-\varsigma)}\left[\int_{0}^{1}\left|\frac{\partial}{\partial \xi} G_{q}(\xi, \tau) \mu(\tau)\right|\right. \\
& +\left(m_{0} \delta^{\beta_{0}}+m_{1} \delta^{\beta_{1}}\right) \int_{0}^{1}\left|\frac{\partial}{\partial \xi} G_{q}(\xi, \tau)\right| \mathrm{d}_{q} \tau \\
& +\frac{2 a \xi}{1-a e^{2}}\left(\int_{0}^{1}\left|G_{q}(\xi, \tau) \mu(\tau)\right| \mathrm{d}_{q} \tau\right. \\
& \left.\left.+\left(m_{0} \delta^{\beta_{0}}+m_{0} \delta^{\beta_{1}}\right) \int_{0}^{1}\left|G_{q}(e, \tau)\right| \mathrm{d}_{q} \tau\right)\right] \mathrm{d}_{q} \xi \\
& \leq \frac{1}{\Gamma_{q}(1-\varsigma)} \int_{0}^{t}(t-\xi)_{q}^{(-\varsigma)}\left(M_{2}+\frac{3}{\Gamma_{q}(\sigma)}\left(m_{0} \delta^{\beta_{0}}+m_{1} \delta^{\beta_{1}}\right)\right) \mathrm{d}_{q} \xi \\
& +\frac{2 a}{\left(1-a e^{2}\right) \Gamma_{q}(1-\varsigma)} \int_{0}^{t} \xi(t-\xi)_{q}^{(-\varsigma)} \\
& \times\left(M_{1}+\frac{2}{\Gamma_{q}(\sigma+1)}\left(m_{0} \delta^{\beta_{0}}+m_{1} \delta^{\beta_{1}}\right)\right) \mathrm{d}_{q} \xi \\
& \leq \frac{1}{\Gamma_{q}(1-\varsigma)}\left(M_{2}+\frac{3}{\Gamma_{q}(\sigma)}\left(m_{0} \delta^{\beta_{0}}+m_{1} \delta^{\beta_{1}}\right)\right) \frac{t^{1-\varsigma}}{1-\varsigma} \\
& +\frac{2 a}{\left(1-a e^{2}\right) \Gamma_{q}(1-\varsigma)}\left(M_{1}+\frac{2}{\Gamma_{q}(\sigma+1)}\left(m_{0} \delta^{\beta_{0}}+m_{1} \delta^{\beta_{1}}\right)\right) \\
& \times \frac{t^{2-\varsigma}}{(1-\varsigma) \Gamma_{q}(2-\varsigma)} \\
& \leq \frac{1}{\Gamma_{q}(2-\varsigma)}\left(M_{2}+\frac{3}{\Gamma_{q}(\sigma)}\left(m_{0} \delta^{\beta_{0}}+m_{1} \delta^{\beta_{1}}\right)\right) \\
& +\frac{2 a}{\left(1-a e^{2}\right) \Gamma_{q}(3-\varsigma)}\left(M_{1}+\frac{2}{\Gamma_{q}(\sigma+1)}\left(m_{0} \delta^{\beta_{0}}+m_{1} \delta^{\beta_{1}}\right)\right) \\
& \leq \frac{3 \Delta}{\Gamma_{q}(2-\varsigma)}\left(m_{0} \delta^{\beta_{0}}\right)+\frac{2 a M_{1}}{\left(1-a e^{2}\right) \Gamma_{q}(2-\varsigma)}+\frac{M_{2}}{\Gamma_{q}(2-\varsigma)} \\
& \leq \frac{1}{2} \delta \text {. }
\end{aligned}
$$

Clearly, $\mathcal{O} y(t)$ and ${ }^{C} \mathcal{D}_{q}^{S}[\mathcal{O} y](t)$ are continuous in $\bar{J}_{0}$. Therefore $\mathcal{O}: \mathcal{B} \rightarrow \mathcal{B}$. In the second case, suppose that condition (A2) holds. Choose

$$
0<\delta \leq \min \left\{\left(\frac{1}{4 \Delta m_{0}}\right)^{\frac{1}{1-\beta_{0}}},\left(\frac{1}{4 \Delta m_{1}}\right)^{\frac{1}{1-\beta_{1}}},\left(\frac{\Gamma_{q}(2-\varsigma)}{6 \Delta m_{0}}\right)^{\frac{1}{1-\beta_{0}}},\left(\frac{\Gamma_{q}(2-\varsigma)}{6 \Delta m_{1}}\right)^{\frac{1}{1-\beta_{1}}}\right\} .
$$

Again, by a similar way, we get $\|\mathcal{O} y\| \leq \delta$, and therefore, in this case, $\mathcal{O}: \mathcal{B} \rightarrow \mathcal{B}$. Here, we need to show that $\mathcal{O}$ is a completely continuous operator. First, the equicontinuity of $\mathcal{O}$ will be shown as follows. Suppose that $s_{1}, s_{2} \in \bar{J}_{0}$ with $s_{1}<s_{2}$ and

$$
N_{0}=1+\max _{t \in \bar{J}_{0}}\left\{\left|w\left(t, y(t),{ }^{C} \mathcal{D}_{q}^{S}[y](t)\right)\right|: y \in \mathcal{B}\right\}
$$


Same et al. Advances in Difference Equations

(2021) 2021:482

Page 9 of 26

Then

$$
\begin{aligned}
\left|\mathcal{O} y\left(s_{1}\right)-\mathcal{O} y\left(s_{2}\right)\right| & \mid \int_{0}^{1}\left(G_{q}\left(s_{2}, \xi\right)-G_{q}\left(s_{1}, \xi\right)\right) w\left(y(\xi),{ }^{C} \mathcal{D}_{q}^{\varsigma}[y](\xi)\right) \mathrm{d}_{q} \xi \\
& +\frac{a\left(s_{2}^{2}-s_{1}^{2}\right)}{1-a e^{2}} \int_{0}^{1} G_{q}(e, \xi) w\left(y(\xi),{ }^{C} \mathcal{D}_{q}^{s}[y](\xi)\right) \mathrm{d}_{q} \xi \mid \\
\leq & N_{0} \int_{0}^{1}\left|G_{q}\left(s_{2}, \xi\right)-G_{q}\left(s_{1}, \xi\right)\right| \mathrm{d}_{q} \xi+\frac{2 a N_{0}}{1-a e^{2}}\left(s_{2}^{2}-s_{1}^{2}\right) \\
\leq & \frac{2 a N_{0}}{1-a e^{2}}\left(s_{2}^{2}-s_{1}^{2}\right)+\frac{N_{0}}{\Gamma_{q}(\sigma)}\left[\int_{0}^{s_{1}}\left(s_{2}^{2}-s_{1}^{2}\right)(1-\xi)_{q}^{(\sigma-1)}\right. \\
& +\left(s_{2}-\xi\right)_{q}^{(\sigma-1)}+\left(s_{1}-\xi\right)_{q}^{(\sigma-1)} \mathrm{d}_{q} \xi \\
& +\int_{s_{1}}^{s_{2}}\left(s_{2}^{2}-s_{1}^{2}\right)(1-\xi)_{q}^{(\sigma-1)}+\left(s_{2}-\xi\right)_{q}^{(\sigma-1)} \mathrm{d}_{q} \xi \\
& \left.+\int_{s_{2}}^{1}\left(s_{2}^{2}-s_{1}^{2}\right)(1-\xi)_{q}^{(\sigma-1)} \mathrm{d}_{q} \xi\right] \\
\leq & \frac{2 a N_{0}}{\left.1-a e^{2}\right)}\left(s_{2}^{2}-s_{1}^{2}\right)+\frac{N_{0}}{\Gamma_{q}(\alpha)}\left[\left(s_{2}^{2}-s_{1}^{2}\right) \int_{0}^{1}(1-\xi)_{q}^{(\sigma-1)} \mathrm{d}_{q} \xi\right. \\
& \left.+\int_{0}^{s_{2}}\left(s_{2}^{2}-\xi\right)_{q}^{(\sigma-1)} \mathrm{d}_{q} \xi-\int_{0}^{s_{1}}\left(s_{1}^{2}-\xi\right)_{q}^{(\sigma-1)} \mathrm{d}_{q} \xi\right] \\
\leq & \frac{N_{0}}{\Gamma_{q}(\alpha+1)}\left[s_{2}^{2}-s_{1}^{2}+s_{2}^{\sigma}-s_{1}^{\sigma}+\frac{2 a\left(s_{2}^{2}-s_{1}^{2}\right)}{1-a e^{2}}\right] \\
\leq & N_{0}\left[\Delta\left(s_{2}^{2}-s_{1}^{2}\right)+\frac{s_{2}^{\sigma}-s_{1}^{\sigma}}{\Gamma_{q}(\sigma+1)}\right],
\end{aligned}
$$

and

$$
\begin{aligned}
\left|{ }^{C} \mathcal{D}_{q}^{\varsigma}[\mathcal{O} y]\left(s_{2}\right)-{ }^{C} \mathcal{D}_{q}^{\varsigma}[\mathcal{O} y]\left(s_{2}\right)\right| & \\
= & \frac{1}{\Gamma_{q}(1-\varsigma)} \mid \int_{0}^{s_{2}}\left(s_{2}-\xi\right)_{q}^{(-\varsigma)}\left(\int_{0}^{1} \frac{\partial}{\partial \xi} G_{q}(\xi, \tau) w\left(y(\tau),{ }^{C} \mathcal{D}_{q}^{\varsigma}[y](\tau)\right) \mathrm{d}_{q} \tau\right. \\
& \left.+\frac{2 a \xi}{1-a e^{2}} \int_{0}^{1} G_{q}(e, \tau) w\left(y(\tau),{ }^{C} \mathcal{D}_{q}^{\varsigma}[y](\tau)\right)\right) \mathrm{d}_{q} \xi \\
& -\int_{0}^{s_{1}}\left(s_{1}-\xi\right)_{q}^{(-\varsigma)}\left(\int_{0}^{1} \frac{\partial}{\partial \xi} G_{q}(\xi, \tau) w\left(y(\tau),{ }^{C} \mathcal{D}_{q}^{\varsigma}[y](\tau)\right) \mathrm{d}_{q} \tau\right. \\
& \left.+\frac{2 a \xi}{1-a e^{2}} \int_{0}^{1} G_{q}(e, \tau) w\left(y(\tau),{ }^{C} \mathcal{D}_{q}^{\varsigma}[y](\tau)\right) \mathrm{d}_{q} \tau\right) \mathrm{d}_{q} \xi \mid \\
\leq & \frac{3 N_{0}}{\Gamma_{q}(1-\varsigma) \Gamma_{q}(\sigma)}\left|\int_{0}^{s_{2}}\left(s_{2}-\xi\right)_{q}^{(-\varsigma)} \mathrm{d}_{q} \xi-\int_{0}^{s_{1}}\left(s_{1}-\xi\right)_{q}^{(-\varsigma)} \mathrm{d}_{q} \xi\right| \\
& +\frac{6 a N_{0}}{\Gamma_{q}(1-\varsigma) \Gamma_{q}(\sigma)\left(1-a e^{2}\right)} \\
& \times\left|\int_{0}^{s_{2}} \xi\left(s_{2}-\xi\right)_{q}^{(-\varsigma)} \mathrm{d}_{q} \xi-\int_{0}^{s_{1}} \xi\left(s_{1}-\xi\right)_{q}^{(-\varsigma)} \mathrm{d}_{q} \xi\right|
\end{aligned}
$$




$$
\begin{aligned}
\leq & \frac{3 N_{0}}{\Gamma_{q}(1-\varsigma) \Gamma_{q}(\sigma)} \mid \int_{0}^{s_{1}}\left(\left(s_{2}-\xi\right)_{q}^{(-\varsigma)}-\left(s_{1}-\xi\right)_{q}^{(-\varsigma)}\right) \mathrm{d}_{q} \xi \\
& +\int_{s_{1}}^{s_{2}}\left(s_{2}-q \xi\right)^{(-\varsigma)} \mathrm{d}_{q} \xi \mid+\frac{6 a N_{0}}{\Gamma_{q}(1-\varsigma) \Gamma_{q}(\sigma)\left(1-a e^{2}\right)} \\
& \times \mid \int_{0}^{s_{1}}\left(\xi\left(s_{2}-\xi\right)_{q}^{(-\varsigma)}-\xi\left(S_{1}-\xi\right)_{q}^{(-\varsigma)}\right) \mathrm{d}_{q} \xi \\
& +\int_{s_{1}}^{s_{2}} \xi\left(s_{2}-q \xi\right)^{(-\varsigma)} \mathrm{d}_{q} \xi \mid \\
\leq & \frac{3 N_{0}}{\Gamma_{q}(1-\varsigma) \Gamma_{q}(\sigma)}\left(s_{2}^{1-\varsigma}-s_{1}^{1-\varsigma}+2\left(s_{2}-s_{1}\right)_{q}^{(1-\varsigma)}\right) \\
& +\frac{6 a N_{0}}{\Gamma_{q}(\sigma)\left(1-a e^{2}\right)} \\
& \times\left(\frac{2 s_{1}\left(s_{2}-s_{1}\right)_{q}^{(1-\varsigma)}}{\Gamma_{q}(2-\varsigma)}+\frac{s_{2}^{2}-s_{1}^{1}}{\Gamma_{q}(3-\varsigma)}+\frac{2\left(s_{2}-s_{1}\right)_{q}^{(2-\varsigma)}}{\Gamma_{q}(3-\varsigma)}\right) .
\end{aligned}
$$

Since the functions $s_{2}^{2}-s_{1}^{2}, d_{2}^{\sigma}-s_{1}^{\sigma},\left(s_{2}-s_{1}\right)_{q}^{(2-\varsigma)}$, and $s_{1}\left(s_{2}-s_{1}\right)^{1-\varsigma}$ are continuous, we conclude that $\mathcal{O} y$ is an equicontinuous set. Obviously, $\mathcal{O} y$ is uniformly bounded because $\mathcal{O}(\mathcal{B}) \subseteq \mathcal{B}$. By means of the Arzelá-Ascoli theorem, $\mathcal{O}$ is a compact operator. Therefore, from the Schauder fixed point theorem, the operator $\mathcal{O}$ has a fixed point, i.e., the $q$-fractional boundary value problem (1) has a solution.

In what follows, we prove the uniqueness of solution for Eq. (1) based on application of the Banach fixed point theorem.

Theorem 3.5 Let $w: \mathbb{R}^{2} \rightarrow \mathbb{R}$ be a continuous function and let it fulfill a Lipschitz condition with respect to the first and second variables with Lipschitz constant

$$
0<\ell<\frac{\Gamma_{q}(2-\varsigma)}{\Delta\left[3+\Gamma_{q}(2-\varsigma)\right]},
$$

i.e.,

$$
\left|w\left(y_{1}, z_{1}\right)-w\left(y_{2}, z_{2}\right)\right| \leq \ell\left(\left|y_{1}-y_{2}\right|+\left|z_{1}-z_{2}\right|\right) .
$$

Then problem (1) has a unique solution.

Proof In Theorem 3.4, we have shown that $\mathcal{O}$ is a continuous operator and $\mathcal{O}: \mathcal{B} \rightarrow \mathcal{B}$. Therefore, using the Banach fixed point theorem, it is sufficient to show that $\mathcal{O}$ is a contraction mapping. For any $y_{1}, y_{2} \in \mathcal{A}$,

$$
\begin{aligned}
& \left|\mathcal{O} y_{1}(t)-\mathcal{O} y_{2}(t)\right| \\
& \leq \mid \int_{0}^{1} G_{q}(t, \xi)\left(w\left(y_{1}(\xi),{ }^{C} \mathcal{D}_{q}^{\varsigma}\left[y_{1}\right](\xi)\right)\right. \\
& \left.\quad-w\left(y_{2}(\xi),{ }^{C} \mathcal{D}_{q}^{\varsigma}\left[y_{2}\right](\xi)\right)\right) \mathrm{d}_{q} \xi \mid \\
& \quad+\frac{a t^{2}}{1-a e^{2}} \mid \int_{0}^{1} G_{q}(e, \xi)\left(w\left(y_{1}(\xi),{ }^{C} \mathcal{D}_{q}^{\varsigma}\left[y_{1}\right](\xi)\right)\right.
\end{aligned}
$$




$$
\begin{aligned}
& \left.-w\left(y_{2}(\xi),{ }^{C} \mathcal{D}_{q}^{S}\left[y_{2}\right](\xi)\right)\right) \mathrm{d}_{q} \xi \\
& \leq \ell\left\|y_{1}-y_{2}\right\|\left(\int_{0}^{1}\left|G_{q}(t, \xi)\right| \mathrm{d}_{q} \xi+\frac{a t^{2}}{1-a e^{2}} \int_{0}^{1}\left|G_{q}(e, \xi)\right| \mathrm{d}_{q} \xi\right) \\
& \leq \ell \Delta\left\|y_{1}-y_{2}\right\|, \\
& \left|{ }^{C} \mathcal{D}_{q}^{\varsigma}[\mathcal{O} y]\left(s_{2}\right)-{ }^{C} \mathcal{D}_{q}^{S}[\mathcal{O} y]\left(s_{2}\right)\right| \\
& =\left|\frac{1}{\Gamma_{q}(1-\varsigma)} \int_{0}^{t}(t-\xi)_{q}^{(-\varsigma)}\left(\mathcal{O}^{\prime} y_{1}(\xi)-\mathcal{O}^{\prime} y_{1}(\xi)\right) \mathrm{d}_{q} \xi\right| \\
& \leq \frac{1}{\Gamma_{q}(1-\varsigma)} \mid \int_{0}^{t}(t-\xi)_{q}^{(-\varsigma)} \\
& \times\left(\int _ { 0 } ^ { 1 } \frac { \partial } { \partial \xi } G _ { q } ( \xi , \tau ) \left(w\left(y_{1}(\tau),{ }^{C} \mathcal{D}_{q}^{\varsigma}\left[y_{1}\right](\tau)\right)\right.\right. \\
& \left.-w\left(y_{2}(\tau),{ }^{C} \mathcal{D}_{q}^{\varsigma}\left[y_{2}\right](\tau)\right)\right) \mathrm{d}_{q} \tau \\
& +\frac{2 a \xi}{1-a e^{2}} \int_{0}^{1} G_{q}(e, \tau)\left(w\left(y_{1}(\tau),{ }^{C} \mathcal{D}_{q}^{\varsigma}\left[y_{1}\right](\tau)\right)\right. \\
& \left.\left.-w\left(y_{2}(\tau),{ }^{C} \mathcal{D}_{q}^{\varsigma}\left[y_{2}\right](\tau)\right)\right) \mathrm{d}_{q} \tau\right) \mathrm{d}_{q} \xi \\
& \leq \frac{3 \ell}{\Gamma_{q}(1-\varsigma) \Gamma_{q}(\sigma)}\left\|y_{1}-y_{2}\right\| \\
& \times\left(\int_{0}^{t}(t-\xi)_{q}^{(-\varsigma)} \mathrm{d}_{q} \xi+\frac{2 a}{1-a e^{2}} \int_{0}^{t} \xi(t-\xi)_{q}^{(-\varsigma)} \mathrm{d}_{q} \xi\right) \\
& \leq \frac{3 \ell \Delta}{\Gamma_{q}(2-\varsigma)}\left\|y_{1}-y_{2}\right\| \text {. }
\end{aligned}
$$

Therefore

$$
\left\|\mathcal{O} y_{1}-\mathcal{O} y_{2}\right\| \leq\left[\Delta \ell+\frac{3 \ell \Delta}{\Gamma_{q}(2-\varsigma)}\right]\left\|y_{1}-y_{2}\right\| .
$$

Hence, by the Banach fixed point theorem, $\mathcal{O}$ has a unique fixed point which is a solution of problem (1).

\subsection{Stability of solution}

In this section, we study the stability analysis of problem (1) under various perturbations. Dependence solution on the boundary value condition is discussed in Theorem 3.6. Stability of the solution with respect to the perturbation of $w$ is analyzed in Theorem 3.7. Finally, the perturbation effect of fractional order derivative on the solution is studied in Lemma 3.8 and Theorem 3.9.

Theorem 3.6 Suppose that function $w$ fulfills the conditions of Theorem 3.5, and let $\hat{v}(t)$ be the solution of the following perturbed problem:

$$
{ }^{C} \mathcal{D}_{q}^{\sigma}[y](t)=w\left(y(t),{ }^{C} \mathcal{D}_{q}^{\varsigma}[y](t)\right)
$$


for each $2<\alpha \leq 3, \varsigma \in J_{0}$, on the boundary value conditions $y(0)=\epsilon_{1}, y^{\prime}(0)=\epsilon_{2}$, and

$$
y(1)=a y(e)+\epsilon_{3}
$$

for $e \in J_{0}$ with $0 \leq a<\frac{1}{e^{2}}$. Then $\|y-\hat{v}\|=O(\epsilon)$, here $\epsilon=\max \left\{\epsilon_{1}, \epsilon_{2}, \epsilon_{3}\right\}$.

Proof Similar to Lemma 2.3 the solution of problem (11) is

$$
\begin{aligned}
\hat{v}(t)= & \int_{0}^{1} G_{q}(t, q \xi) w\left(\hat{v}(\xi),{ }^{C} \mathcal{D}_{q}^{S}[\hat{v}(\xi)]\right) \mathrm{d}_{q} \xi \\
& +\frac{a t^{2}}{1-a e^{2}} \int_{0}^{1} G_{q}(e, q \xi) w\left(\hat{v}(\xi),{ }^{C} \mathcal{D}_{q}^{s}[\hat{v}](\xi)\right) \mathrm{d}_{q} \xi+h(t)
\end{aligned}
$$

where

$$
h(t)=\frac{t^{2}}{1-a e^{2}}\left(\epsilon_{1}(a-1)+\epsilon_{2}(a e-1)\right)+\epsilon_{2} t+\epsilon_{1} .
$$

Thus,

$$
\begin{aligned}
|y-\hat{v}| \leq & \left|\int_{0}^{1} G_{q}(t, \xi)\left[w\left(y(\xi),{ }^{C} \mathcal{D}_{q}^{s}[y](\xi)\right)-w\left(y(\xi),{ }^{C} \mathcal{D}_{q}^{s}[y](\xi)\right)\right] \mathrm{d}_{q} \xi\right| \\
& +\frac{a t^{2}}{1-a e^{2}} \mid \int_{0}^{1} G_{q}(e, \xi)\left[w\left(y(\xi),{ }^{C} \mathcal{D}_{q}^{s}[y](\xi)\right)\right. \\
& \left.-w\left(\hat{v}(\xi),{ }^{C} \mathcal{D}_{q}^{\varsigma}[\hat{v}](\xi)\right)\right] \mathrm{d}_{q} \xi|+| h(t) \mid \\
\leq & \ell\|y-\hat{v}\|\left(\int_{0}^{1} G_{q}(t, \xi) \mathrm{d}_{q} \xi+\frac{a t^{2}}{1-a e^{2}} \int_{0}^{1} G_{q}(t, \xi) \mathrm{d}_{q} \xi\right)+|h(t)| \\
\leq & \ell \Delta\|y-\hat{v}\|+|h(t)|,
\end{aligned}
$$

and

$$
\begin{aligned}
&\left|{ }^{C} \mathcal{D}_{q}^{\varsigma}[y](t)-{ }^{C} \mathcal{D}_{q}^{\varsigma}[\hat{v}](t)\right| \\
&=\frac{1}{\Gamma_{q}(1-\varsigma)} \mid \int_{0}^{t}(t-\xi)_{q}^{(-\varsigma)} \\
& \times\left(\int_{0}^{1} \frac{\partial}{\partial \xi} G_{q}(\xi, \tau)\left(w\left(y(\tau),{ }^{C} \mathcal{D}_{q}^{\varsigma}[y](\tau)\right)-w\left(\hat{v}(\tau),{ }^{C} \mathcal{D}_{q}^{\varsigma}[\hat{v}](\tau)\right)\right) \mathrm{d}_{q} \tau\right. \\
&+\frac{2 a \xi}{1-a e^{2}} \int_{0}^{1} G_{q}(e, \tau)\left(w\left(y(\tau),{ }^{C} \mathcal{D}_{q}^{\varsigma}[y](\tau)\right)\right. \\
&\left.\left.-w\left(\hat{v}(\tau),{ }^{C} \mathcal{D}_{q}^{\varsigma}[\hat{v}](\tau)\right)\right) \mathrm{d}_{q} \tau\right) \mathrm{d}_{q} \xi|+|{ }^{C} \mathcal{D}_{q}^{\varsigma}[h](t) \mid \\
& \leq \frac{3 \ell}{\Gamma_{q}(1-\varsigma) \Gamma_{q}(\sigma)}\|y-\hat{v}\|\left(\int_{0}^{t}(t-\xi)_{q}^{(-\varsigma)} \mathrm{d}_{q} \xi\right. \\
&\left.+\frac{2 a \xi}{1-a e^{2}} \int_{0}^{t} \xi(t-\xi)_{q}^{(-\varsigma)} \mathrm{d}_{q} \xi\right)+\left|{ }^{C} \mathcal{D}_{q}^{\varsigma}[h](t)\right| \\
& \leq \frac{3 \ell \Delta}{\Gamma_{q}(1-\varsigma)}\|y-\hat{v}\|+\left|{ }^{C} \mathcal{D}_{q}^{\varsigma}[h](t)\right|
\end{aligned}
$$


Therefore,

$$
\begin{aligned}
\|y-\hat{v}\| \leq & \frac{1}{1-\left(\ell \Delta+\frac{3 \ell \Delta}{\Gamma_{q}(2-\varsigma)}\right)} \\
& \times\left(\left|\frac{a t^{2}}{1-a e^{2}}\left(\epsilon_{1}(a-1)+\epsilon_{2}(a e-1)\right)+\epsilon_{2} t+\epsilon_{1}\right|\right. \\
& \left.+\left|\frac{2 t^{2-\varsigma}}{\left(1-a e^{2}\right) \Gamma_{q}(3-\varsigma)}\left(\epsilon_{1}(a-1)+\epsilon_{2}(a e-1)\right)+\frac{\epsilon_{2}}{\Gamma_{q}(2-\varsigma)} t^{1-\varsigma}\right|\right) \\
\leq & \frac{\epsilon}{1-\left(\ell \Delta+\frac{3 \ell \Delta}{\Gamma_{q}(2-\varsigma)}\right)} \\
& \times\left|\frac{1}{1-a e^{2}}\left[1+\frac{2}{\Gamma_{q}(3-\varsigma)}\right](a+2 a e)+2+\frac{1}{\Gamma_{q}(2-\varsigma)}\right| .
\end{aligned}
$$

This completes the proof.

Theorem 3.7 Suppose that the conditions of Theorem 3.5 hold, and let $\hat{v}(t)$ be the solution of the following perturbed problem on function $w$ :

$$
{ }^{C} \mathcal{D}_{q}^{\alpha}[y](t)=w\left(y(t),{ }^{C} \mathcal{D}_{q}^{\varsigma}[y](t)\right)+\epsilon
$$

for $t \in \bar{J}_{0}, 2<\alpha \leq 3$, and $\varsigma \in J_{0}$, with the boundary conditions $y_{0}=y_{0}^{\prime}=0, y_{1}=a y(e)$ for $e \in J_{0}$ with $0 \leq a<\frac{1}{e^{2}}$. Then $\|y-\hat{v}\|=O(\epsilon)$.

Proof The solution of problem (13) is

$$
\begin{aligned}
\hat{v}(t)= & \int_{0}^{1} G_{q}(t, \xi)\left(w\left(\hat{v}(\xi),{ }^{C} \mathcal{D}_{q}^{\varsigma}\right)+\epsilon\right) \mathrm{d}_{q} \xi \\
& +\frac{a t^{2}}{1-a e^{2}} \int_{0}^{1} G_{q}(e, \xi)\left(w\left(\hat{v}(\xi),{ }^{C} \mathcal{D}_{q}^{\varsigma}[\hat{v}](\xi)\right)+\epsilon\right) \mathrm{d}_{q} \xi .
\end{aligned}
$$

Then, similar to the proof of the previous theorem

$$
\begin{aligned}
|y-\hat{v}| & \leq \ell \Delta\|y-\hat{v}\|+\epsilon\left(\int_{0}^{1} G_{q}(t, \xi) \mathrm{d}_{q} \xi+\frac{a t^{2}}{1-a e^{2}} \int_{0}^{1} G_{q}(t, \xi) \mathrm{d}_{q} \xi\right) \\
& \leq \Delta\|y-\hat{v}\|+\epsilon \Delta
\end{aligned}
$$

and

$$
\begin{aligned}
\left|{ }^{C} \mathcal{D}_{q}^{\varsigma}[y](t)-{ }^{C} \mathcal{D}_{q}^{\varsigma}[\hat{v}](t)\right| \leq & \frac{3 \ell \Delta}{\Gamma_{q}(2-\varsigma) \Gamma_{q}(\sigma)}\|y-\hat{v}\| \\
& +\epsilon\left(\int_{0}^{t}(t-\xi)_{q}^{(-\varsigma)} \mathrm{d}_{q} \xi+\frac{2 a}{1-a e^{2}} \int_{0}^{t} \xi(t-\xi)_{q}^{(-\varsigma)} \mathrm{d}_{q} \xi\right) \\
\leq & \frac{3 \ell \Delta}{\Gamma_{q}(2-\varsigma)}\|y-\hat{v}\|+\frac{3 \epsilon \Delta}{\Gamma_{q}(2-\varsigma)} .
\end{aligned}
$$


Indeed,

$$
\|y-\hat{v}\| \leq \frac{\epsilon}{1-\left(\ell \Delta+\frac{3 \epsilon \Delta}{\Gamma_{q}(2-\zeta)}\right)}\left[\Delta+\frac{3 \Delta}{\Gamma_{q}(2-\varsigma)}\right] .
$$

This completes the proof.

For perturbation analysis on the fractional order of the $q$-derivative, we first state and prove the following lemma and then the main theorem will be discussed.

Lemma 3.8 Let $s, t \in \bar{J}_{0}$ and $2<\sigma-\epsilon<\sigma$, then

$$
\int_{0}^{t}\left|\frac{s^{\sigma-1}}{\Gamma_{q}(\sigma)}-\frac{s^{\sigma-\epsilon-1}}{\Gamma_{q}(\sigma-\epsilon)}\right| \mathrm{d}_{q} s=O(\epsilon)
$$

Proof We estimate the integral as follows:

$$
\begin{aligned}
\int_{0}^{t}\left|\frac{s^{\sigma-1}}{\Gamma_{q}(\sigma)}-\frac{s^{\sigma-\epsilon-1}}{\Gamma_{q}(\sigma-\epsilon)}\right| \mathrm{d}_{q} s \leq & \int_{0}^{t}\left|\frac{s^{\sigma-1}}{\Gamma_{q}(\sigma)}-\frac{s^{\sigma-\epsilon-1}}{\Gamma_{q}(\sigma-\epsilon)}\right| \mathrm{d}_{q} s \\
& +\int_{0}^{t}\left|\frac{s^{\sigma-\epsilon-1}}{\Gamma_{q}(\sigma)}-\frac{s^{\sigma-\epsilon-1}}{\Gamma_{q}(\sigma-\epsilon)}\right| \mathrm{d}_{q} s \\
\leq & \frac{1}{\Gamma_{q}(\sigma)}\left[\frac{1}{\sigma}-\frac{1}{\alpha-\epsilon}\right]+\frac{1}{\sigma-\epsilon}\left[\frac{1}{\Gamma_{q}(\sigma)}-\frac{1}{\Gamma_{q}(\sigma-\epsilon)}\right] \\
\leq & \epsilon\left[\frac{1}{\sigma(\sigma-\epsilon) \Gamma_{q}(\sigma)}+\frac{\left|\Gamma_{q}(\alpha)\right|}{(\sigma-\epsilon) \Gamma_{q}(\sigma)(\sigma-\epsilon)}\right],
\end{aligned}
$$

where $\sigma-\epsilon<\alpha<\sigma$.

Theorem 3.9 Suppose that the conditions of Theorem 3.5 hold, and let $\hat{v}(t)$ be the solution of the following perturbed problem on fractional order derivative $\sigma$ :

$$
{ }^{C} \mathcal{D}_{q}^{\sigma-\epsilon}[y](t)=w\left(y(t),{ }^{C} \mathcal{D}_{q}^{\zeta}[y](t)\right),
$$

for $t \in \bar{J}_{0}, 2<\sigma \leq 3, \varsigma \in J_{0}$, under the boundary conditions $y_{0}=y_{0}^{\prime}=0, y_{1}=a y(e), e \in J_{0}$ with $0 \leq a<\frac{1}{e^{2}}$ and $2<\sigma-\epsilon<\sigma \leq 3$. Then $\|y-\hat{v}\|=O(\epsilon)$.

Proof According to the above discussion, the solution of problem (15) is given by

$$
\begin{aligned}
\hat{v}(t)= & \int_{0}^{1} \hat{G}_{q}(t, \xi) w\left(\hat{v}(\xi),{ }^{C} \mathcal{D}_{q}^{s}[\hat{v}](\xi)\right) \mathrm{d}_{q} \xi \\
& +\frac{a t^{2}}{1-a e^{2}} \int_{0}^{1} \hat{G}_{q}(t, \xi) w\left(\hat{v}(\xi),{ }^{C} \mathcal{D}_{q}^{s}[\hat{v}](\xi)\right) \mathrm{d}_{q} \xi,
\end{aligned}
$$

where

$$
\hat{G}_{q}(t, \xi)= \begin{cases}\frac{(t-\xi)_{q}^{(\sigma-\epsilon-1)}-t^{2}(1-\xi)_{q}^{(\sigma-\epsilon-1)}}{\Gamma_{q}(\sigma)}, & \xi<t, \\ \frac{-t^{2}(1-\xi)_{q}^{(\sigma-\epsilon-1)}}{\Gamma_{q}(\sigma)}, & t<\xi,\end{cases}
$$


for $t, \xi \in \bar{J}_{0}$. Then

$$
\begin{aligned}
& |y-\hat{v}| \leq \mid \int_{0}^{1} G_{q}(t, \xi) w\left(y(\xi),{ }^{C} \mathcal{D}_{q}^{\varsigma}[y](\xi)\right) \mathrm{d}_{q} \xi \\
& -\int_{0}^{1} \hat{G}_{q}(t, \xi) w\left(\hat{v}(\xi),{ }^{C} \mathcal{D}_{q}^{\varsigma}[\hat{v}](\varsigma)\right) \mathrm{d}_{q} \xi \\
& +\frac{a t^{2}}{1-a e^{2}} \mid \int_{0}^{1} G_{q}(e, \xi) w\left(y(\xi),{ }^{C} \mathcal{D}_{q}^{\varsigma}[y](\xi)\right) \mathrm{d}_{q} \xi \\
& -\int_{0}^{1} \hat{G}_{q}(e, \xi) w\left(\hat{v}(\xi),{ }^{C} \mathcal{D}_{q}^{s}[\hat{v}](\xi)\right) \mathrm{d}_{q} \xi \\
& \leq\left|\int_{0}^{1} G_{q}(t, \xi)\left(w\left(y(\xi),{ }^{C} \mathcal{D}_{q}^{\varsigma}[y](\xi)\right)-w\left(\hat{v}(\xi),{ }^{C} \mathcal{D}_{q}^{\varsigma}[\hat{v}]\right)(\xi)\right) \mathrm{d}_{q} \xi\right| \\
& +\left|\int_{0}^{1}\left(G_{q}(e, \xi)-\hat{G}_{q}(e, \xi)\right) w\left(\hat{v}(\xi),{ }^{C} \mathcal{D}_{q}^{S}[\hat{v}](\xi)\right) \mathrm{d}_{q} \xi\right| \\
& +\frac{a t^{2}}{1-a e^{2}}\left(\mid \int_{0}^{1} G_{q}(e, \xi)\left(w\left(y(\xi),{ }^{C} \mathcal{D}_{q}^{s}[y](\xi)\right)\right.\right. \\
& \left.-w\left(\hat{v}(\xi),{ }^{C} \mathcal{D}_{q}^{\varsigma}[\hat{v}](\xi)\right)\right) \mathrm{d}_{q} \xi \\
& \left.+\left|\int_{0}^{1}\left(G_{q}(e, \xi)-\hat{G}_{q}(e, \xi)\right) w\left(\hat{v}(\xi),{ }^{C} \mathcal{D}_{q}^{\zeta}[\hat{v}](\xi)\right)\right|\right) \\
& \leq \ell\|y-\hat{v}\| \int_{0}^{1}\left|G_{q}(t, \xi) \mathrm{d}_{q} \xi+\|w\|_{\epsilon} \int_{0}^{1}\right| G_{q}(t, \xi)-\hat{G}_{q}(t, \xi) \mid \mathrm{d}_{q} \xi \\
& +\frac{a}{1-a e^{2}}\left(\ell\|y-\hat{v}\| \int_{0}^{1}\left|G_{q}(e, \xi)\right| \mathrm{d}_{q} \xi\right. \\
& \left.+\|w\|_{\epsilon} \int_{0}^{1}\left|G_{q}(e, \xi)-\hat{G}_{q}(e, \xi)\right| \mathrm{d}_{q} \xi\right) \\
& \leq \ell \Delta\|y-\hat{v}\|+\|w\|_{\epsilon}\left(\int_{0}^{1}\left|G_{q}(t, \xi)-\hat{G}_{q}(t, \xi)\right| \mathrm{d}_{q} \xi\right. \\
& \left.+\frac{a}{1-a e^{2}} \int_{0}^{1}\left|G_{q}(e, \xi)-\hat{G}_{q}(e, \xi)\right| \mathrm{d}_{q} \xi\right),
\end{aligned}
$$

where

$$
\|w\|_{\epsilon}=\sup _{0<\epsilon<\sigma-2}\left|w\left(\hat{v}(t),{ }^{C} \mathcal{D}_{q}^{\varsigma}[\hat{v}](t)\right)\right| .
$$

Also, we have

$$
\begin{aligned}
\left|{ }^{C} \mathcal{D}_{q}^{\varsigma}[y](t)-{ }^{C} \mathcal{D}_{q}^{\varsigma}[\hat{v}](t)\right| \leq & \frac{1}{\Gamma_{q}(1-\varsigma)} \mid \int_{0}^{t}(t-\xi)_{q}^{(-\varsigma)} \\
& \times\left(\int_{0}^{1} \frac{\partial}{\partial \xi} G_{q}(\xi, \tau) w\left(y(\tau),{ }^{C} \mathcal{D}_{q}^{\varsigma}[y](\tau)\right)\right. \\
& \left.-\int_{0}^{1} \frac{\partial}{\partial \xi} \hat{G}_{q}(\xi, \tau) w\left(\hat{v}(\tau),{ }^{C} \mathcal{D}_{q}^{\varsigma}[\hat{v}](\tau)\right) \mathrm{d}_{q} \tau\right) \mathrm{d}_{q} \xi
\end{aligned}
$$


Same et al. Advances in Difference Equations

(2021) 2021:482

Page 16 of 26

$$
\begin{aligned}
& +\frac{2 a}{\Gamma_{q}(1-\varsigma)\left(1-a e^{2}\right)} \mid \int_{0}^{t}(t-\xi)_{q}^{(-\varsigma)} \\
& \times\left(\int_{0}^{1} \xi G_{q}(e, \tau) w\left(y(\tau),{ }^{C} \mathcal{D}_{q}^{\varsigma}[y](\tau)\right)\right. \\
& \left.-\int_{0}^{1} \xi \hat{G}(e, \tau) w\left(\hat{v}(\tau),{ }^{C} \mathcal{D}_{q}^{S}[\hat{v}](\tau)\right) \mathrm{d}_{q} \tau\right) \mathrm{d}_{q} \xi \\
& \leq \frac{\ell\|y-\hat{v}\|}{\Gamma_{q}(1-\varsigma)} \int_{0}^{t}(t-\xi)_{q}^{(-\varsigma)}\left(\int_{0}^{1}\left|\frac{\partial}{\partial \xi} G_{q}(\xi, \tau)\right| \mathrm{d}_{q} \tau\right) \mathrm{d}_{q} \xi \\
& +\|w\|_{\epsilon} \frac{1}{\Gamma_{q}(1-\varsigma)} \int_{0}^{t}(t-\xi)_{q}^{(-\varsigma)} \\
& \times\left(\int_{0}^{1}\left|\frac{\partial}{\partial \xi} G_{q}(\xi, \tau)-\frac{\partial}{\partial \xi} \hat{G}_{q}(\xi, \tau)\right| \mathrm{d}_{q} \tau\right) \mathrm{d}_{q} \xi \\
& +\frac{2 a}{\Gamma_{q}(1-\varsigma)\left(1-a e^{2}\right)}[\ell\|y-\hat{v}\| \\
& \times \int_{0}^{t} \xi(t-\xi)_{q}^{(-\varsigma)}\left(\int_{0}^{1}\left|G_{q}(e, \tau)\right| \mathrm{d}_{q} \tau\right) \mathrm{d}_{q} \xi \\
& +\|w\|_{\epsilon} \int_{0}^{t} \xi(t-\xi)_{q}^{(-\varsigma)} \\
& \left.\times\left(\int_{0}^{1}\left|G_{q}(e, \tau)-\hat{G}_{q}(e, \tau)\right| \mathrm{d}_{q} \tau\right) \mathrm{d}_{q} \xi\right] \\
& \leq \frac{3 \ell \Delta}{\Gamma_{q}(2-\varsigma)}\|y-\hat{v}\|+\|\ell\|_{\epsilon} \frac{1}{\Gamma_{q}(1-\varsigma)} \int_{0}^{t} \xi(t-\xi)_{q}^{(-\varsigma)} \\
& \times\left(\int_{0}^{1}\left|\frac{\partial}{\partial \xi} G_{q}(\xi, \tau)-\frac{\partial}{\partial \xi} \hat{G}_{q}(\xi, \tau)\right| \mathrm{d}_{q} \tau\right) \mathrm{d}_{q} \xi \\
& +\frac{2 a}{\Gamma_{q}(1-\varsigma)\left(1-a e^{2}\right)}\|w\|_{\epsilon} \\
& \times \int_{0}^{t} \xi(t-\xi)_{q}^{(-\varsigma)}\left(\int_{0}^{1}\left|G_{q}(e, \tau)-\hat{G}_{q}(e, \tau)\right| \mathrm{d}_{q} \tau\right) \mathrm{d}_{q} \xi .
\end{aligned}
$$

Therefore,

$$
\begin{aligned}
\|y-\hat{v}\| \leq & \frac{1}{1-\left(\ell \Delta+\frac{3 \ell \Delta}{\Gamma_{q}(2-\varsigma)}\right)}\left[\int_{0}^{t}\left|G_{q}(t, \xi)-\hat{G}_{q}(t, \xi)\right| \mathrm{d}_{q} \xi\right. \\
& +\frac{a}{1-a e^{2}} \int_{0}^{1}\left|G_{q}(e, \xi)-\hat{G}_{q}(e, \xi)\right| \mathrm{d}_{q} \xi \\
& +\frac{1}{\Gamma_{q}(1-\varsigma)} \int_{0}^{t} \xi(t-q \xi)^{(-\varsigma)} \\
& \times\left(\int_{0}^{1}\left|\frac{\partial}{\partial \xi} G_{q}(\xi, \tau)-\frac{\partial}{\partial \xi} \hat{G}_{q}(\xi, \tau)\right| \mathrm{d}_{q} \tau\right) \mathrm{d}_{q} \xi \\
& +\frac{2 a}{\Gamma_{q}(1-\varsigma)\left(1-a e^{2}\right)} \int_{0}^{t} \xi(t-q \xi)^{(-\varsigma)} \\
& \left.\times\left(\int_{0}^{t}\left|G_{q}(e, \tau)-\hat{G}_{q}(e, \tau)\right| \mathrm{d}_{q} \tau\right) \mathrm{d}_{q} \xi\right] .
\end{aligned}
$$


According to the structure of $G_{q}(t, \xi)$, we know that every term of $\left|G_{q}(t, \xi)-\hat{G}_{q}(t, \xi)\right|$ and

$$
\left|\frac{\partial}{\partial \xi} G_{q}(t, \xi)-\frac{\partial}{\partial \xi} \hat{G}_{q}(t, \xi)\right|
$$

is in the form of Eq. (15). Hence, Lemma 3.8 implies

$$
\begin{aligned}
& \int_{0}^{1}\left|G_{q}(t, \xi)-\hat{G}_{q}(t, \xi)\right| \mathrm{d}_{q} \xi=O(\epsilon), \\
& \int_{0}^{1}\left|\frac{\partial}{\partial \xi} G_{q}(t, \xi)-\frac{\partial}{\partial \xi} \hat{G}_{q}(t, \xi)\right|=O(\epsilon) .
\end{aligned}
$$

Therefore, $\|y-\hat{v}\|=O(\epsilon)$ and the proof is complete.

\section{Some illustrative examples}

Herein, we give some examples to show the validity of the main results. In this way, we give a computational technique for checking problem (1). We need to present a simplified analysis that is able to execute the values of the $q$-gamma function. For this purpose, we provided a pseudo-code description of the method for calculation of the $q$-gamma function of order $n$ [61].

Example 4.1 Consider the problem

$$
{ }^{D} \mathcal{D}_{q}^{\frac{8}{3}}[y](t)=\frac{4}{7}(y(t))^{\frac{1}{2}}+\frac{3}{10}\left({ }^{C} \mathcal{D}_{q}^{\frac{1}{2}}[y](t)\right)^{\frac{1}{4}}
$$

via boundary conditions $y(0)=y^{\prime}(0)=0$ and $y(1)=\frac{14}{9} y\left(\frac{3}{5}\right)$. Clearly, $\sigma=\frac{8}{3} \in(2,3], \varsigma=\frac{1}{2} \in$ $J_{0}, e=\frac{3}{5} \in \bar{J}_{0}$, and $a=\frac{14}{9} \in\left[0, \frac{1}{e^{2}}\right)$. We define $w: \mathbb{R}^{2} \rightarrow \mathbb{R}$ by

$$
w(y, z)=\frac{4}{7}(y(t))^{\frac{1}{2}}+\frac{3}{10}(z(t))^{\frac{1}{4}}
$$

for $y, z \in \mathbb{R}$. Then we have

$$
\begin{aligned}
\left|w\left(y(t),{ }^{C} \mathcal{D}_{q}^{\frac{1}{2}}[y](t)\right)\right| & =\left|\frac{4}{7}(y(t))^{\frac{1}{2}}+\frac{3}{10}\left({ }^{C} \mathcal{D}_{q}^{\frac{1}{2}}[z](t)\right)^{\frac{1}{4}}\right| \\
& \leq \frac{4}{7}\left|(y(t))^{\frac{1}{2}}\right|+\frac{3}{10}\left|\left({ }^{C} \mathcal{D}_{q}^{\frac{1}{2}}[z](t)\right)^{\frac{1}{4}}\right| \\
& \leq \mu(t)+\frac{4}{7}\left|(y(t))^{\frac{1}{2}}\right|+\frac{3}{10}\left|\left({ }^{C} \mathcal{D}_{q}^{\frac{1}{2}}[z](t)\right)^{\frac{1}{4}}\right|,
\end{aligned}
$$

where $\mu(t)=\exp (t)$. We take $m_{0}=\frac{4}{7}, m_{1}=\frac{3}{10}, \beta_{0}=\frac{1}{2}$, and $\beta_{1}=\frac{1}{4}$. Also, by using Eq. (8), we obtain

$$
\begin{aligned}
\Delta & =\frac{2}{\Gamma_{q}(\sigma+1)}\left[1+\frac{a}{1-a e^{2}}\right] \\
& =\frac{2}{\Gamma_{q}\left(\frac{8}{3}+1\right)}\left[1+\frac{14}{9\left(1-\frac{14}{25}\right)}\right]=\frac{2}{\Gamma_{q}\left(\frac{11}{3}\right)} \times \frac{449}{99}=\frac{898}{99 \Gamma_{q}\left(\frac{11}{3}\right)}
\end{aligned}
$$


Table 1 Numerical results of $\Gamma_{q}(\sigma+1)$ and $\Delta$ for $q=\frac{1}{5}, \frac{1}{2}, \frac{7}{8}$ in Example 4.1 (Algorithm 1)

\begin{tabular}{|c|c|c|c|c|c|c|}
\hline \multirow[t]{2}{*}{$n$} & \multicolumn{2}{|l|}{$q=\frac{1}{5}$} & \multicolumn{2}{|l|}{$q=\frac{1}{2}$} & \multicolumn{2}{|l|}{$q=\frac{7}{8}$} \\
\hline & $\overline{\Gamma_{q}(\sigma+1)}$ & $\Delta$ & $\overline{\Gamma_{q}(\sigma+1)}$ & $\Delta$ & $\overline{\Gamma_{q}(\sigma+1)}$ & $\Delta$ \\
\hline 1 & 1.3971 & $6.4927 \mathrm{E}+00$ & 2.6906 & $3.3713 \mathrm{E}+00$ & 4.1775 & $2.1713 \mathrm{E}-01$ \\
\hline 2 & 1.3860 & $6.5444 E+00$ & 2.4015 & $3.7771 \mathrm{E}+00$ & 2.5979 & $3.4916 \mathrm{E}-01$ \\
\hline 3 & 1.3839 & $6.5547 \mathrm{E}+00$ & 2.2738 & $3.9892 \mathrm{E}+00$ & 1.8239 & 4.9733E-01 \\
\hline 4 & 1.3834 & $6.5568 \mathrm{E}+00$ & 2.2136 & $4.0976 \mathrm{E}+00$ & 1.3865 & $6.5422 \mathrm{E}-01$ \\
\hline 5 & $\underline{1.3833}$ & $6.5572 \mathrm{E}+00$ & 2.1844 & 4.1524E+00 & 1.1146 & $8.1380 E-01$ \\
\hline 6 & 1.3833 & $\underline{6.5573 \mathrm{E}+00}$ & 2.1700 & 4.1800E+00 & 9.3373 & $9.7145 \mathrm{E}-01$ \\
\hline 7 & 1.3833 & $\overline{6.5573 E+00}$ & 2.1629 & $4.1938 \mathrm{E}+00$ & 8.0715 & $1.1238 \mathrm{E}+00$ \\
\hline \multirow[t]{2}{*}{8} & 1.3833 & $6.5573 E+00$ & 2.1593 & $4.2007 E+00$ & 7.1505 & $1.2685 E+00$ \\
\hline & $\vdots$ & $\vdots$ & : & $\vdots$ & & \\
\hline 13 & 1.3833 & $6.5573 E+00$ & 2.1559 & 4.2074E+00 & 4.9118 & $1.8467 E+00$ \\
\hline 14 & 1.3833 & $6.5573 E+00$ & 2.1558 & 4.2075E+00 & 4.6925 & $1.9330 E+00$ \\
\hline 15 & 1.3833 & $6.5573 E+00$ & $\overline{2.1558}$ & $\underline{4.2076 \mathrm{E}+00}$ & 4.5115 & $2.0106 \mathrm{E}+00$ \\
\hline 16 & 1.3833 & $6.5573 E+00$ & 2.1558 & $\overline{4.2076 \mathrm{E}+00}$ & 4.3610 & $2.0800 E+00$ \\
\hline \multirow[t]{2}{*}{17} & 1.3833 & $6.5573 \mathrm{E}+00$ & 2.1558 & $4.2076 \mathrm{E}+00$ & 4.2349 & $2.1419 E+00$ \\
\hline & : & : & : & : & & \\
\hline 75 & 1.3833 & $6.5573 E+00$ & 2.1558 & $4.2076 \mathrm{E}+00$ & 3.4790 & $2.6073 \mathrm{E}+00$ \\
\hline 76 & 1.3833 & $6.5573 E+00$ & 2.1558 & $4.2076 \mathrm{E}+00$ & 3.4789 & $2.6073 \mathrm{E}+00$ \\
\hline 77 & 1.3833 & $6.5573 E+00$ & 2.1558 & $4.2076 \mathrm{E}+00$ & $\overline{3.4789}$ & $2.6074 \mathrm{E}+00$ \\
\hline 78 & 1.3833 & $6.5573 E+00$ & 2.1558 & $4.2076 \mathrm{E}+00$ & 3.4789 & $2.6074 \mathrm{E}+00$ \\
\hline
\end{tabular}

and

$$
\begin{aligned}
\delta \geq & \max \left\{\left(6 \Delta m_{0}\right)^{\frac{1}{1-\beta_{0}}},\left(6 \Delta m_{1}\right)^{\frac{1}{1-\beta_{1}}}, 6 \Delta M_{1},\left(\frac{12 \Delta m_{0}}{\Gamma_{q}(2-\varsigma)}\right)^{\frac{1}{1-\beta_{0}}},\right. \\
& \left.\left(\frac{12 \Delta m_{1}}{\Gamma_{q}(2-\varsigma)}\right)^{\frac{1}{1-\beta_{1}}}, \frac{16 a M_{1}}{\Gamma_{q}(2-\varsigma)\left(1-a e^{2}\right)}, \frac{8 M_{2}}{\Gamma_{q}(2-\varsigma)}\right\} \\
= & \max \left\{\left(\frac{24}{7} \Delta\right)^{2},\left(\frac{9}{5} \Delta\right)^{\frac{4}{3}}, 6 \Delta M_{1},\left(\frac{48 \Delta}{7 \Gamma_{q}\left(\frac{3}{2}\right)}\right)^{2},\left(\frac{18 \Delta}{5 \Gamma_{q}\left(\frac{3}{2}\right)}\right)^{\frac{4}{3}},\right. \\
& \left.\frac{5600 M_{1}}{99 \Gamma_{q}\left(\frac{3}{2}\right)}, \frac{8 M_{2}}{\Gamma_{q}\left(\frac{3}{2}\right)}\right\} .
\end{aligned}
$$

Table 1 shows $\Delta \cong 6.5573,4.2076,2.6074$ for $q=\frac{1}{5}, \frac{1}{2}, \frac{7}{8}$, respectively. Figure 1 shows $2 \mathrm{D}$ graphs of $\Delta$. Therefore, condition (A1) in Theorem 3.4 holds, and hence this problem has a solution.

Example 4.2 Consider the following problem:

$$
{ }^{C} \mathcal{D}_{q}^{\frac{27}{\Pi 1}}[y](t)=\frac{4}{5}(y(t))^{3}+3\left({ }^{C} \mathcal{D}_{q}^{\frac{1}{8}}[y](t)\right)^{4}
$$

under the boundary conditions $y(0)=y^{\prime}(0)=0$ and $y(1)=\frac{1}{2} y\left(\frac{2}{7}\right)$. Then

$$
w\left(y(t),{ }^{C} \mathcal{D}_{q}^{\frac{2}{5}}[y](t)\right) \leq 4|y(t)|^{3}+2\left|{ }^{C} \mathcal{D}_{q}^{\frac{2}{5}}[y](t)\right|^{5} .
$$

Clearly, $\sigma=\frac{27}{11} \in(2,3], \varsigma=\frac{1}{8} \in J_{0}, e=\frac{2}{7} \in \bar{J}_{0}$, and $a=\frac{19}{4} \in\left[0, \frac{1}{e^{2}}\right)$. We define $w: \mathbb{R}^{2} \rightarrow \mathbb{R}$ by

$$
w(y, z)=\frac{4}{5}(y(t))^{3}+3(z(t))^{4}
$$




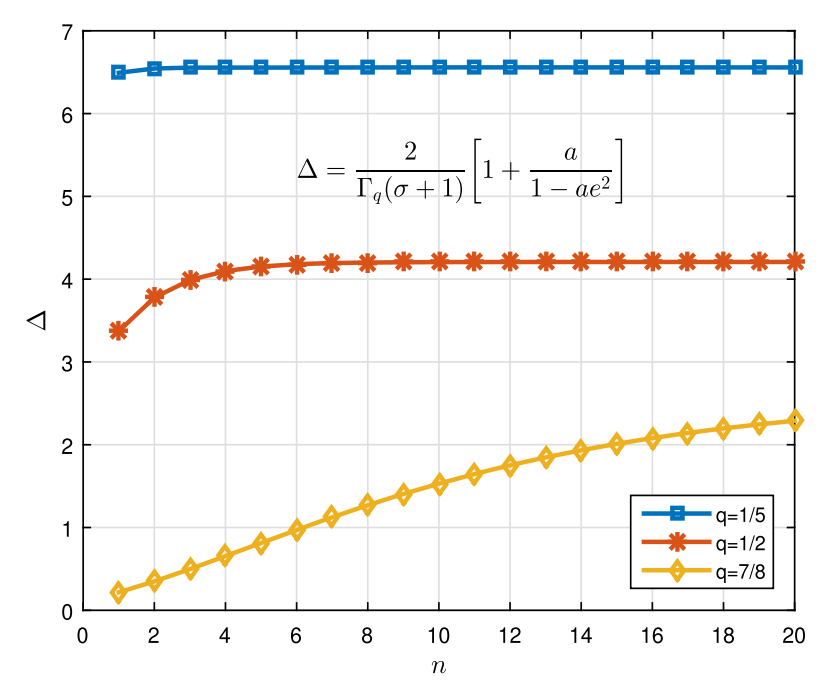

Figure $12 \mathrm{D}$ graphs of $\Delta$ for $q=\frac{1}{5}, \frac{1}{2}, \frac{7}{8}$ in Example 4.1

for $y, z \in \mathbb{R}$. Then we have

$$
\begin{aligned}
\left|w\left(y(t),{ }^{C} \mathcal{D}_{q}^{\frac{1}{8}}[y](t)\right)\right| & =\frac{4}{5}\left|(y(t))^{3}\right|+3\left({ }^{C} \mathcal{D}_{q}^{\frac{1}{8}}[y](t)\right)^{4} \\
& \leq \frac{4}{5}|y(t)|^{3}+3\left|{ }^{C} \mathcal{D}_{q}^{\frac{1}{8}}[y](t)\right|^{4} .
\end{aligned}
$$

We take $m_{0}=\frac{4}{5}, m_{1}=3, \beta_{0}=3$, and $\beta_{1}=4$. Also, by using Eq. (8), we obtain

$$
\begin{aligned}
\Delta & =\frac{2}{\Gamma_{q}(\sigma+1)}\left[1+\frac{a}{1-a e^{2}}\right] \\
& =\frac{2}{\Gamma_{q}\left(\frac{27}{11}+1\right)}\left[1+\frac{19}{4\left(1-\frac{19}{49}\right)}\right] \\
& =\frac{2}{\Gamma_{q}\left(\frac{38}{11}\right)} \times \frac{1051}{120}=\frac{2102}{120 \Gamma_{q}\left(\frac{38}{11}\right)} .
\end{aligned}
$$

Table 2 shows $\Delta \cong 1.3258,9.1665 \times 10^{1}, 6.2138$ for $q=\frac{1}{5}, \frac{1}{2}, \frac{7}{8}$, respectively. Figure 2 shows 2D graphs of $\Delta$. Therefore, condition (A2) in Theorem 3.4 holds, and hence this problem has a solution.

Example 4.3 Consider the problem

$$
{ }^{C} \mathcal{D}_{q}^{\frac{12}{5}}[y](t)=\frac{1}{18}(y(t))+\frac{1}{9} \sin \left({ }^{C} \mathcal{D}_{q}^{\frac{3}{7}}[y](t)\right)
$$

with boundary conditions $y(0)=y^{\prime}(0)=0$ and $y(1)=\frac{1}{3} y\left(\frac{8}{11}\right)$. It is clear that $\sigma=\frac{12}{5} \in(2,3]$, $\varsigma=\frac{3}{7} \in J_{0}, e=\frac{8}{11} \in \bar{J}_{0}$, and $a=\frac{81}{64} \in\left[0, \frac{1}{e^{2}}\right)$. We define $w: \mathbb{R}^{2} \rightarrow \mathbb{R}$ by

$$
w(y, z)=\frac{1}{18} y(t)+\frac{1}{9} \sin (z(t))
$$


Table 2 Numerical results of $\Gamma_{q}(\sigma+1)$ and $\Delta$ for $q=\frac{1}{5}, \frac{1}{2}, \frac{7}{8}$ in Example 4.2 (Algorithm 1)

\begin{tabular}{|c|c|c|c|c|c|c|}
\hline \multirow[t]{2}{*}{$n$} & \multicolumn{2}{|l|}{$q=\frac{1}{5}$} & \multicolumn{2}{|l|}{$q=\frac{1}{2}$} & \multicolumn{2}{|l|}{$q=\frac{7}{8}$} \\
\hline & $\Gamma_{q}(\sigma+1)$ & $\Delta$ & $\Gamma_{q}(\sigma+1)$ & $\Delta$ & $\Gamma_{q}(\sigma+1)$ & $\Delta$ \\
\hline 1 & 1.3343 & $1.3128 \mathrm{E}+01$ & 2.3699 & $7.3912 \mathrm{E}+00$ & 29.1233 & $6.0147 \mathrm{E}-01$ \\
\hline 2 & 1.3238 & $1.3232 \mathrm{E}+01$ & 2.1221 & $8.2545 E+00$ & 18.5831 & $9.4262 \mathrm{E}-01$ \\
\hline 3 & 1.3217 & $1.3253 E+01$ & 2.0124 & $8.7044 \mathrm{E}+00$ & 13.3129 & $1.3158 \mathrm{E}+00$ \\
\hline 4 & 1.3213 & $1.3257 \mathrm{E}+01$ & 1.9607 & $8.9339 E+00$ & 10.2860 & $1.7030 E+00$ \\
\hline 5 & $\underline{1.3212}$ & $1.3258 \mathrm{E}+01$ & 1.9356 & $9.0499 \mathrm{E}+00$ & 8.3794 & $2.0905 E+00$ \\
\hline \multirow[t]{2}{*}{6} & $\overline{1.3212}$ & $\overline{1.3258 E+01}$ & 1.9232 & $9.1081 E+00$ & 7.0969 & $2.4682 \mathrm{E}+00$ \\
\hline & $\vdots$ & : & $\vdots$ & : & & \\
\hline 14 & 1.3212 & $1.3258 \mathrm{E}+01$ & 1.9110 & $9.1663 E+00$ & 3.7276 & $4.6992 \mathrm{E}+00$ \\
\hline 15 & 1.3212 & $1.3258 \mathrm{E}+01$ & 1.9110 & $9.1665 E+00$ & 3.5932 & $4.8750 E+00$ \\
\hline 16 & 1.3212 & $1.3258 \mathrm{E}+01$ & $\underline{1.9109}$ & $9.1665 \mathrm{E}+00$ & 3.4811 & $5.0320 E+00$ \\
\hline \multirow[t]{2}{*}{17} & 1.3212 & $1.3258 \mathrm{E}+01$ & $\overline{1.9109}$ & $\overline{9.1665 E+00}$ & 3.3870 & $5.1717 E+00$ \\
\hline & $\vdots$ & $\vdots$ & $\vdots$ & i & : & \\
\hline 81 & 1.3212 & $1.3258 \mathrm{E}+01$ & 1.9109 & $9.1666 \mathrm{E}+00$ & 2.8191 & $6.2137 E+00$ \\
\hline 82 & 1.3212 & $1.3258 \mathrm{E}+01$ & 1.9109 & $9.1666 \mathrm{E}+00$ & 2.8190 & $6.2137 E+00$ \\
\hline 83 & 1.3212 & $1.3258 \mathrm{E}+01$ & 1.9109 & $9.1666 \mathrm{E}+00$ & $\overline{2.8190}$ & $6.2137 E+00$ \\
\hline 84 & 1.3212 & $1.3258 \mathrm{E}+01$ & 1.9109 & $9.1666 \mathrm{E}+00$ & 2.8190 & $6.2138 \mathrm{E}+00$ \\
\hline 85 & 1.3212 & $1.3258 \mathrm{E}+01$ & 1.9109 & $9.1666 \mathrm{E}+00$ & 2.8190 & $\overline{6.2138 \mathrm{E}+00}$ \\
\hline 86 & 1.3212 & $1.3258 \mathrm{E}+01$ & 1.9109 & $9.1666 \mathrm{E}+00$ & 2.8190 & $6.2138 \mathrm{E}+00$ \\
\hline 87 & 1.3212 & $1.3258 \mathrm{E}+01$ & 1.9109 & $9.1666 \mathrm{E}+00$ & 2.8190 & $6.2138 \mathrm{E}+00$ \\
\hline
\end{tabular}

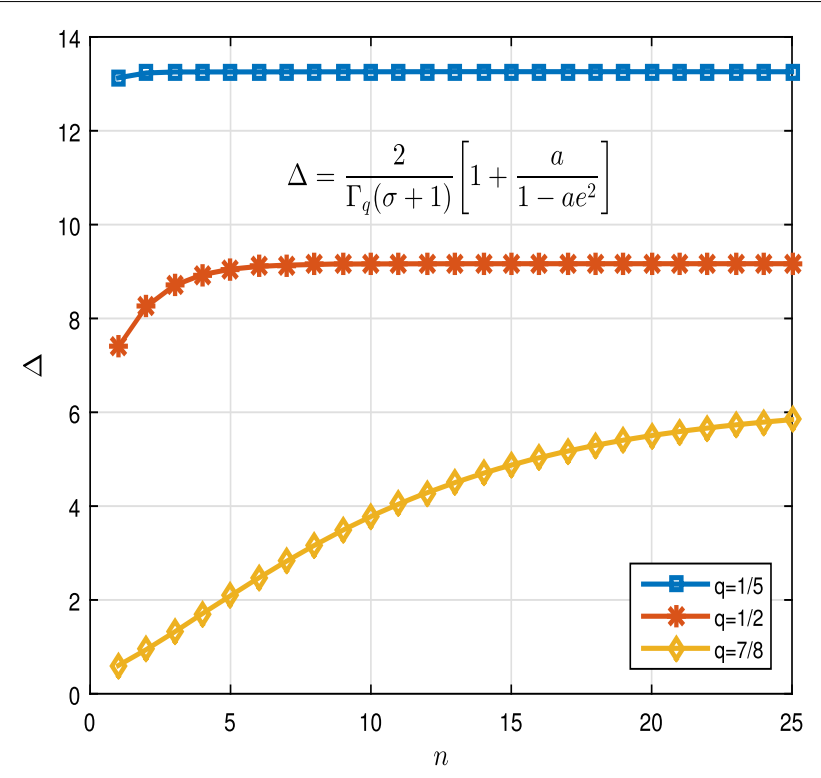

Figure 2 2D graphs of $\Delta$ for $q=\frac{1}{5}, \frac{1}{2}, \frac{7}{8}$ in Example 4.2

for $y, z \in \mathbb{R}$. Then we have

$$
\begin{aligned}
\left|w\left(y(t),{ }^{C} \mathcal{D}_{q}^{\frac{3}{7}}[y](t)\right)-w\left(z(t),{ }^{C} \mathcal{D}_{q}^{\frac{3}{7}}[z](t)\right)\right| \\
=\mid \frac{1}{18}(y(t))+\frac{1}{9} \sin \left({ }^{C} \mathcal{D}_{q}^{\frac{3}{7}}[y](t)\right) \\
\quad-\left(\frac{1}{18}(z(t))+\frac{1}{9} \sin \left({ }^{C} \mathcal{D}_{q}^{\frac{3}{7}}[z](t)\right)\right) \mid
\end{aligned}
$$




$$
\begin{aligned}
\leq & \frac{1}{18}|y(t)-z(t)| \\
& +\frac{1}{9}\left|\sin \left({ }^{C} \mathcal{D}_{q}^{\frac{3}{7}}[y](t)\right)-\sin \left({ }^{C} \mathcal{D}_{q}^{\frac{3}{7}}[z](t)\right)\right| \\
\leq & \frac{1}{18}|y(t)-z(t)|+\frac{1}{9}\left|{ }^{C} \mathcal{D}_{q}^{\frac{3}{7}}[y](t)-{ }^{C} \mathcal{D}_{q}^{\frac{3}{7}}[z](t)\right| \\
\leq & \frac{1}{9}\left(|y(t)-z(t)|+\left|{ }^{C} \mathcal{D}_{q}^{\frac{3}{7}}[y](t)-{ }^{C} \mathcal{D}_{q}^{\frac{3}{7}}[z](t)\right|\right) .
\end{aligned}
$$

We take $\ell=\frac{1}{9}$. Table 3 shows that

\begin{tabular}{|c|c|c|c|}
\hline$n$ & $\Gamma_{q}(\sigma+1)$ & $\Delta$ & $\ell$ \\
\hline & $q=\frac{1}{5}$ & & \\
\hline 1 & 1.3187 & $7.3233 E+00$ & $1.3655 \mathrm{E}-01$ \\
\hline 2 & 1.3084 & $7.3811 \mathrm{E}+00$ & $1.3548 \mathrm{E}-01$ \\
\hline 3 & 1.3063 & $7.3927 \mathrm{E}+00$ & $1.3527 \mathrm{E}-01$ \\
\hline 4 & 1.3059 & $7.3950 \mathrm{E}+00$ & $1.3523 \mathrm{E}-01$ \\
\hline 5 & 1.3058 & $7.3954 \mathrm{E}+00$ & $1.3522 \mathrm{E}-01$ \\
\hline \multirow[t]{2}{*}{6} & 1.3058 & $7.3955 E+00$ & $1.3522 \mathrm{E}-01$ \\
\hline & $q=\frac{1}{2}$ & & \\
\hline 1 & 2.2951 & $4.2077 \mathrm{E}+00$ & $2.3766 \mathrm{E}-01$ \\
\hline 2 & 2.0569 & 4.6949E+00 & $2.1300 \mathrm{E}-01$ \\
\hline 3 & 1.9515 & $4.9486 \mathrm{E}+00$ & $2.0208 \mathrm{E}-01$ \\
\hline & & & $\vdots$ \\
\hline 10 & 1.8546 & $5.2070 \mathrm{E}+00$ & $1.9205 \mathrm{E}-01$ \\
\hline 11 & 1.8543 & $5.2080 E+00$ & $1.9201 \mathrm{E}-01$ \\
\hline 12 & 1.8541 & $5.2086 \mathrm{E}+00$ & $1.9199 \mathrm{E}-01$ \\
\hline 13 & 1.8540 & $5.2088 \mathrm{E}+00$ & $1.9198 \mathrm{E}-01$ \\
\hline 14 & 1.8539 & $5.2089 E+00$ & $1.9198 \mathrm{E}-01$ \\
\hline 15 & 1.8539 & $5.2090 \mathrm{E}+00$ & $1.9198 \mathrm{E}-01$ \\
\hline \multirow[t]{2}{*}{16} & 1.8539 & $5.2090 \mathrm{E}+00$ & $1.9197 \mathrm{E}-01$ \\
\hline & $q=\frac{7}{8}$ & & \\
\hline 1 & 26.5678 & $3.6349 \mathrm{E}-01$ & $2.7511 E+00$ \\
\hline 2 & 17.0689 & 5.6577E-01 & $1.7675 \mathrm{E}+00$ \\
\hline 3 & 12.2939 & $7.8552 \mathrm{E}-01$ & $1.2731 E+00$ \\
\hline 4 & 9.5395 & $1.0123 E+00$ & $9.8783 \mathrm{E}-01$ \\
\hline \multirow[t]{2}{*}{5} & 7.7985 & $1.2383 \mathrm{E}+00$ & $8.0755 \mathrm{E}-01$ \\
\hline & & $\vdots$ & \\
\hline 80 & 2.6759 & $3.6089 \mathrm{E}+00$ & $2.7709 \mathrm{E}-01$ \\
\hline 81 & 2.6759 & $\underline{3.6090 \mathrm{E}+00}$ & $2.7709 \mathrm{E}-01$ \\
\hline 82 & 2.6758 & $\overline{3.6090 E+00}$ & $2.7709 E-01$ \\
\hline 83 & 2.6758 & $3.6090 \mathrm{E}+00$ & $2.7709 \mathrm{E}-01$ \\
\hline 84 & 2.6758 & $3.6090 \mathrm{E}+00$ & $2.7709 \mathrm{E}-01$ \\
\hline 85 & 2.6758 & $3.6090 \mathrm{E}+00$ & $2.7708 \mathrm{E}-01$ \\
\hline 86 & 2.6758 & $3.6090 \mathrm{E}+00$ & $\overline{2.7708 \mathrm{E}-01}$ \\
\hline 87 & 2.6758 & $3.6090 \mathrm{E}+00$ & $2.7708 \mathrm{E}-01$ \\
\hline 88 & 2.6758 & $3.6090 \mathrm{E}+00$ & $2.7708 \mathrm{E}-01$ \\
\hline
\end{tabular}

$$
\frac{\Gamma_{q}(2-\varsigma)}{\Delta\left[3+\Gamma_{q}(2-\varsigma)\right]}=1.3522 \times 10^{-1}, 1.9197 \times 10^{-1}, 2.7708 \times 10^{-1}
$$

Table 3 Numerical results of $\Gamma_{q}(\sigma+1), \Delta$, and $\ell<\frac{\Gamma_{q}(2-\varsigma)}{\Delta\left[3+\Gamma_{q}(2-\varsigma)\right]}$ for $q=\frac{1}{5}, \frac{1}{2}, \frac{7}{8}$ in Example 4.2 (Algorithm 2) 


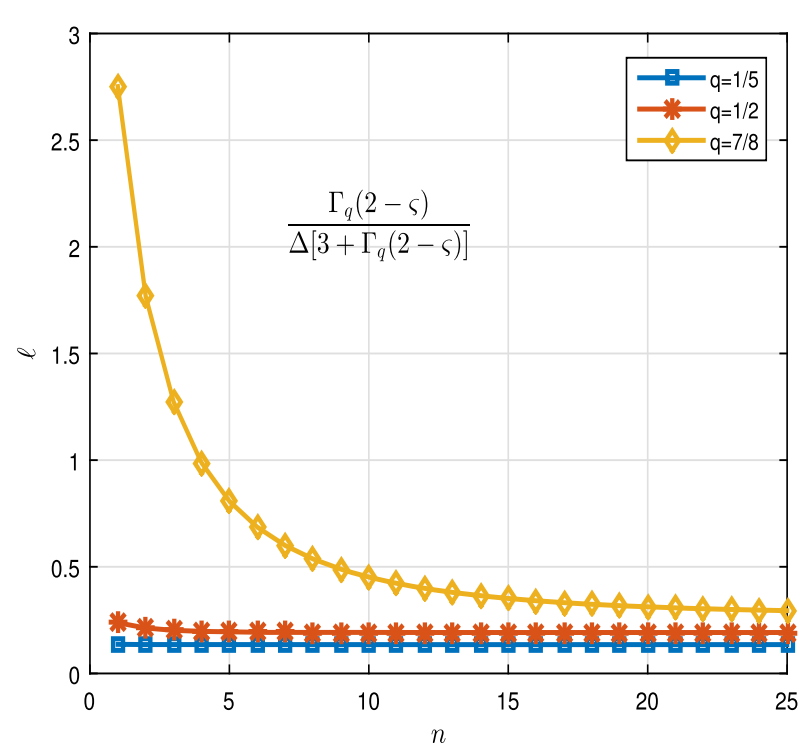

Figure 32 graphs of $\frac{\Gamma_{q}(2-\varsigma)}{\Delta\left[3+\Gamma_{q}(2-\varsigma)\right]}$ for $q=\frac{1}{5}, \frac{1}{2}, \frac{7}{8}$ in Example 4.3

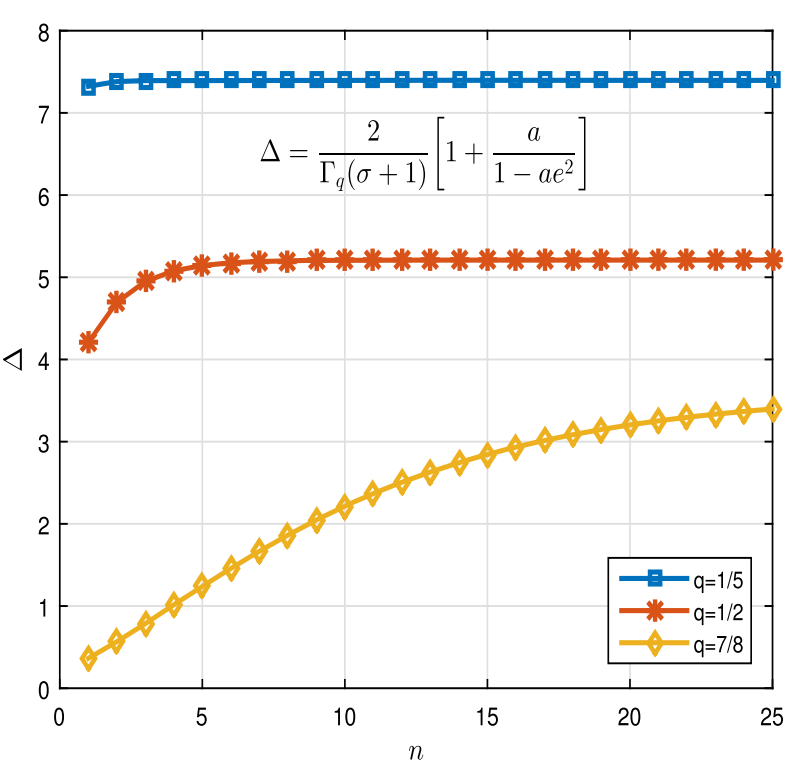

Figure 4 2D graphs of $\Delta$ for $q=\frac{1}{5}, \frac{1}{2}, \frac{7}{8}$ in Example 4.3

for $q=\frac{1}{5}, \frac{1}{2}, \frac{7}{8}$, respectively. Also, the results prove that

$$
\ell \leq \frac{\Gamma_{q}(2-\varsigma)}{\Delta\left[3+\Gamma_{q}(2-\varsigma)\right]}
$$

Figure 3 shows 2D graphs of

$$
\frac{\Gamma_{q}(2-\varsigma)}{\Delta\left[3+\Gamma_{q}(2-\varsigma)\right]}
$$


Also, by using Eq. (8), we obtain

$$
\begin{aligned}
\Delta & =\frac{2}{\Gamma_{q}(\sigma+1)}\left[1+\frac{a}{1-a e^{2}}\right] \\
& =\frac{2}{\Gamma_{q}\left(\frac{12}{5}+1\right)}\left[1+\frac{81}{64\left(1-\frac{81}{121}\right)}\right]=\frac{2}{\Gamma_{q}\left(\frac{17}{5}\right)} \times \frac{12,361}{2560}=\frac{24,722}{2560 \Gamma_{q}\left(\frac{38}{11}\right)} .
\end{aligned}
$$

Table 3 shows that $\Delta=7.3955,5.2090,3.6090$ for $q=\frac{1}{5}, \frac{1}{2}, \frac{7}{8}$, respectively. Figure 4 shows $2 \mathrm{D}$ graphs of $\Delta$. Now, by applying Eq. (10), we get

$$
\ell<\frac{\Gamma_{q}(2-\varsigma)}{\Delta\left[3+\Gamma_{q}(2-\varsigma)\right]}=\frac{\Gamma_{q}\left(2-\frac{3}{7}\right)}{\Delta\left[3+\Gamma_{q}\left(2-\frac{3}{7}\right)\right]}=\frac{\Gamma_{q}\left(\frac{11}{7}\right)}{\Delta\left[3+\Gamma_{q}\left(\frac{11}{7}\right)\right]} .
$$

Since $0<\ell<\frac{1}{9}<0.263$, Theorem 3.5 implies that this problem has a unique solution.

\section{Conclusion}

The Schauder fixed point theorem has been applied in the research study to discuss the well-posed conditions for a class of $q$-fractional order boundary value problems As a result, we have proved the existence and uniqueness of solution by means of the Schauder fixed point and Banach contraction map theorems on the interval $[0,1]$. We have also studied the perturbation on boundary condition on the function exists in the right-hand side of the problem and on the fractional order. To the leading of our information, the results have never been detailed in other works $[11,12,61]$ that consider the problems. In this manner, it is very apparent that the solution of the problem is stable under the small perturbation.

\section{Appendix: Supporting information}

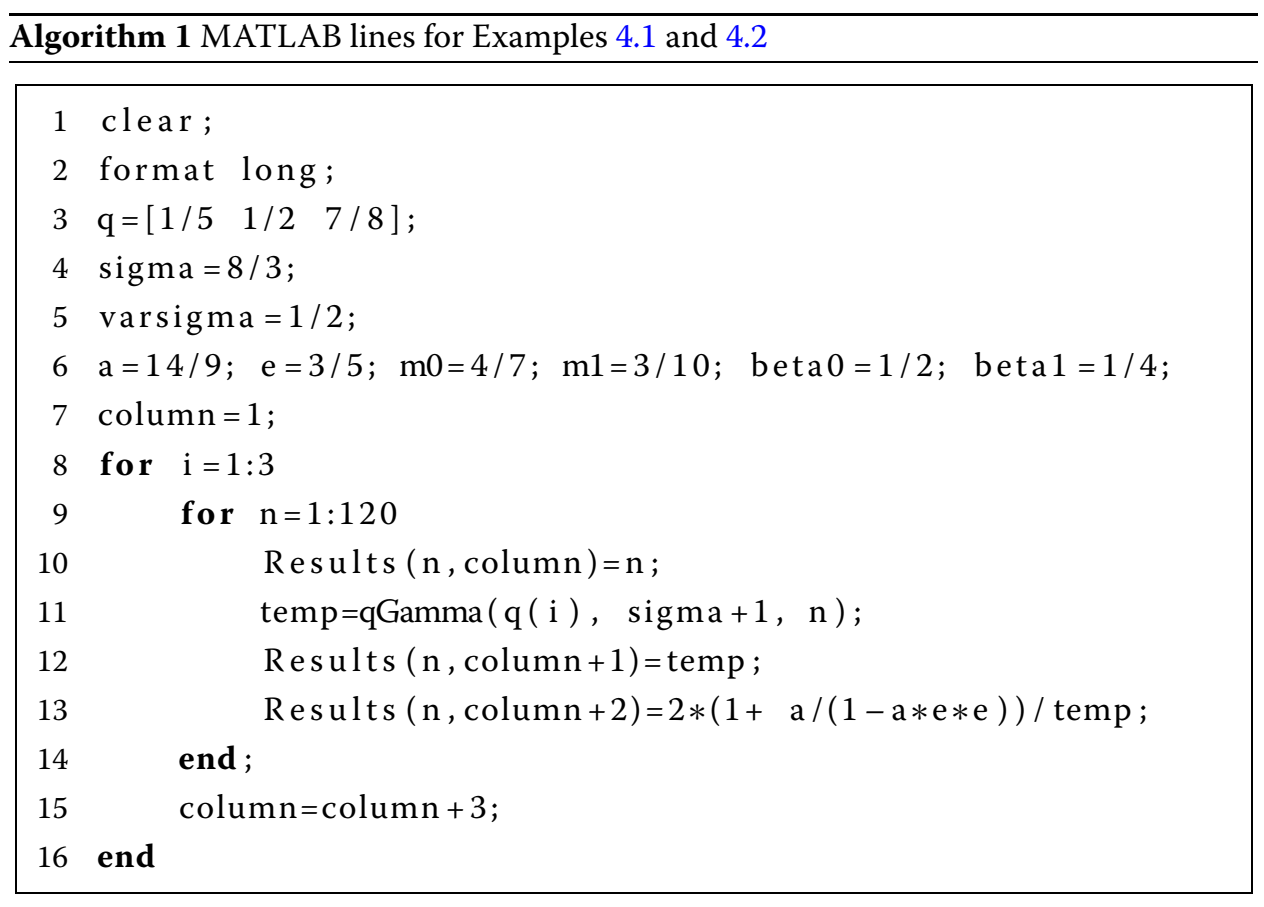




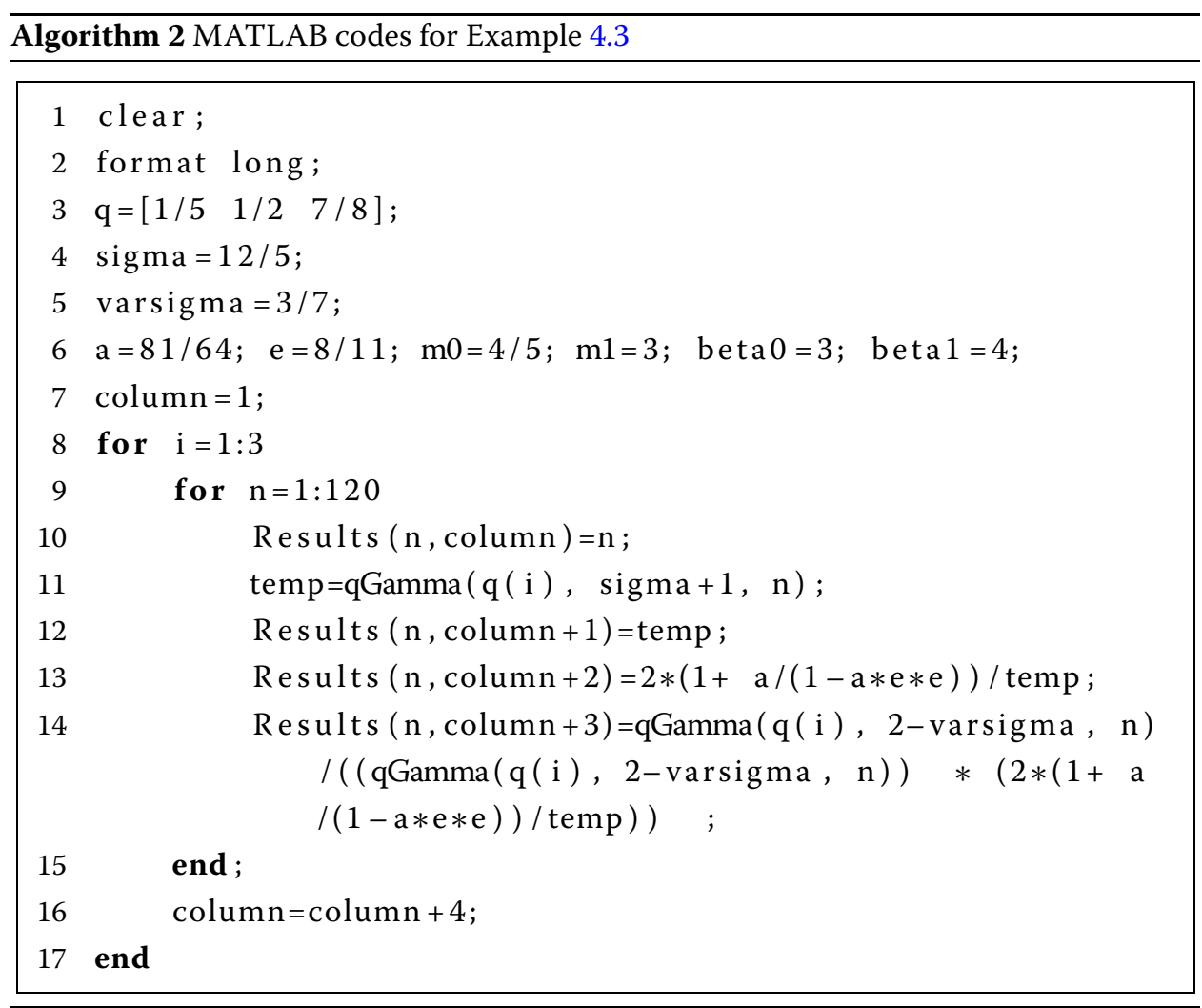

\section{Acknowledgements}

The first and second authors were supported by Bu-Ali Sina University. The fifth author was supported by Azarbaijan Shahid Madani University. The authors express their gratitude to dear unknown referees for their helpful suggestions which improved the final version of this paper.

\section{Funding}

Not applicable.

\section{Availability of data and materials}

Data sharing not applicable to this article as no datasets were generated or analyzed during the current study.

\section{Declarations}

Ethics approval and consent to participate

Not applicable.

\section{Consent for publication}

Not applicable.

\section{Competing interests}

The authors declare that they have no competing interests.

\section{Authors' contributions}

The authors declare that the study was realized in collaboration with equal responsibility. All authors read and approved the final manuscript.

\section{Author details}

'Department of Mathematics, Bu-Ali Sina University, 65178, Hamedan, Iran. ${ }^{2}$ Department of Mathematics, Sacred Heart College (Autonomous), Tirupattur, 635 601, Tamil Nadu, India. ${ }^{3}$ Department of Mathematics and General Sciences, Prince Sultan University, Riyadh, Saudi Arabia. ${ }^{4}$ Department of Industrial Engineering, OSTiM Technical University, 06374 Ankara, Turkey. ${ }^{5}$ Department of Mathematics, Azarbaijan Shahid Madani University, Tabriz, Iran. ${ }^{6}$ Department of Medical Research, China Medical University Hospital, China Medical University, Taichung, Taiwan.

\section{Publisher's Note}

Springer Nature remains neutral with regard to jurisdictional claims in published maps and institutional affiliations. 
Received: 30 August 2021 Accepted: 13 October 2021 Published online: 06 November 2021

\section{References}

1. Kilbas, A.A., Srivastava, H.M., Trujillo, J.J.: Theory and Applications of Fractional Differential Equations. North-Holland Mathematics Studies. Elsevier, Amsterdam (2006)

2. Podlubny, I.: Fractional Differential Equations. Academic Press, New York (1993)

3. Rezapour, S., Imran, A., Hussain, A., Martinez, F., Etemad, S., Kaabar, M.K.A.: Condensing functions and approximate endpoint criterion for the existence analysis of quantum integro-difference FBVPs. Symmetry 13(3), 469 (2021). https://doi.org/10.3390/sym13030469

4. Adiguzel, R.S., Aksoy, U., Karapinar, E., Erhan, I.M.: On the solutions of fractional differential equations via Geraghty type hybrid contractions. Appl. Comput. Math. 20(2), 313-333 (2021)

5. Adiguzel, R.S., Aksoy, U., Karapinar, E., Erhan, I.M.: Uniqueness of solution for higher-order nonlinear fractional differential equations with multi-point and integral boundary conditions. Rev. R. Acad. Cienc. Exactas Fís. Nat., Ser. A Mat. 2021, 155 (2021). https://doi.org/10.1007/s13398-021-01095-3

6. Alqahtani, B., Aydi, H., Karapinar, E., Rakocevic, V.: A solution for Volterra fractional integral equations by hybrid contractions. Mathematics 7(8), 694 (2019). https://doi.org/10.3390/math7080694

7. Bachir, F.S., Abbas, S., Benbachir, M., Benchohra, M.: Hilfer-Hadamard fractional differential equations; existence and attractivity. Adv. Theory Nonlinear Anal. Appl. 5, 49-57 (2021)

8. Baitiche, Z., Derbazi, C., Benchohra, M.: $\psi$-Caputo fractional differential equations with multi-point boundary conditions by topological degree theory. Results Nonlinear Anal. 3, 167-178 (2020)

9. Baleanu, D., Jajarmi, A., Mohammadi, H., Rezapour, S.: A new study on the mathematical modelling of human liver with Caputo-Fabrizio fractional derivative. Chaos Solitons Fractals 134, 109705 (2020). https://doi.org/10.1016/j.chaos.2020.109705

10. Karapinar, E., Fulga, A., Rashid, M., Shahid, L., Aydi, H.: Large contractions on quasi-metric spaces with an application to nonlinear fractional differential equations. Mathematics 7(5), 444 (2019). https://doi.org/10.3390/math7050444

11. Abdeljawad, A., Agarwal, R.P., Karapinar, E., Kumari, P.S.: Solutions of the nonlinear integral equation and fractional differential equation using the technique of a fixed point with a numerical experiment in extended $b$-metric space. Symmetry 11, 686 (2019). https://doi.org/10.3390/sym11050686

12. Adiguzel, R.S., Aksoy, U., Karapinar, E., Erhan, I.M.: On the solution of a boundary value problem associated with a fractional differential equation. Math. Methods Appl. Sci. (2020). https://doi.org/10.1002/mma.6652

13. Karapinar, E., Fulga, A.: An admissible hybrid contraction with an Ulam type stability. Demonstr. Math. 52, 428-436 (2019). https://doi.org/10.1515/dema-2019-0037

14. Alqahtani, B., Fulga, A., Karapinar, E.: Fixed point results on $\delta$-symmetric quasi-metric space via simulation function with an application to Ulam stability. Mathematics 6(10), 208 (2018). https://doi.org/10.3390/math6100208

15. Brzdek, J., Karapinar, E., Petrsel, A.: A fixed point theorem and the Ulam stability in generalized dq-metric spaces. J. Math. Anal. Appl. 467, 501-520 (2018). https://doi.org/10.1016/j.jmaa.2018.07.022

16. Rezapour, S., Azzaoui, B., Tellab, B., Etemad, S., Masiha, H.P.: An analysis on the positive solutions for a fractional configuration of the Caputo multiterm semilinear differential equation. J. Funct. Spaces 2021, Article ID 6022941 (2021). https://doi.org/10.1155/2021/6022941

17. Matar, M.M., Abbas, M.I., Alzabut, J., Kaabar, M.K.A., Etemad, S., Rezapour, S.: Investigation of the p-Laplacian nonperiodic nonlinear boundary value problem via generalized Caputo fractional derivatives. Adv. Differ. Equ. 2021, 68 (2021). https://doi.org/10.1186/s13662-021-03228-9

18. Baleanu, D., Rezapour, S., Saberpour, Z:: On fractional integro-differential inclusions via the extended fractional Caputo-Fabrizio derivation. Bound. Value Probl. 2019, 79 (2019). https://doi.org/10.1186/s13661-019-1194-0

19. Baleanu, D., Etemad, S., Rezapour, S.: A hybrid Caputo fractional modeling for thermostat with hybrid boundary value conditions. Bound. Value Probl. 2020, 64 (2020). https://doi.org/10.1186/s13661-020-01361-0

20. Jackson, F.H.: q-Difference equations. Am. J. Math. 32, 305-314 (1910). https://doi.org/10.2307/2370183

21. Al-Salam, W.A.: q-Analogues of Cauchy's formula. Proc. Am. Math. Soc. 17, 182-184 (1952-1953)

22. Agarwal, R.P.: Certain fractional q-integrals and q-derivatives. Proc. Camb. Philos. Soc. 66, 365-370 (1965). https://doi.org/10.1017/S0305004100045060

23. Adams, C.R.: The general theory of a class of linear partial q-difference equations. Trans. Am. Math. Soc. 26, 283-312 (1924)

24. Annaby, M.H., Mansour, Z.S.: q-Fractional Calculus and Equations. Springer, Cambridge (2012). https://doi.org/10.1007/978-3-642-30898-7

25. Abdeljawad, T., Alzabut, J., Baleanu, D.: A generalized $q$-fractional Gronwall inequality and its applications to nonlinear delay $q$-fractional difference systems. J. Inequal. Appl. 2016, 240 (2016). https://doi.org/10.1186/s13660-016-1181-2

26. Ahmad, B., Etemad, S., Ettefagh, M., Rezapour, S.: On the existence of solutions for fractional $q$-difference inclusions with q-antiperiodic boundary conditions. Bull. Math. Soc. Sci. Math. Roum. 59(107(2)), 119-134 (2016). https://doi.org/10.1186/s13660-016-1181-2

27. Goodrich, C.S.: Existence of a positive solution to a class of fractional differential equations. Appl. Math. Lett. 23, 1050-1055 (2010)

28. Jafari, H., Daftardar-Gejji, V.: Positive solutions of nonlinear fractional boundary value problems using Adomian decomposition method. Appl. Math. Comput. 180(2), 700-706 (2006). https://doi.org/10.1186/s13662-020-02766-y

29. Ferreira, R.A.C.: Nontrivial solutions for fractional $q$-difference boundary value problems. Electron. J. Qual. Theory Differ. Equ. 2010, 70 (2010)

30. Samei, M.E., Rezapour, S.: On a system of fractional $q$-differential inclusions via sum of two multi-term functions on a time scale. Bound. Value Probl. 2020, 135 (2020). https://doi.org/10.1186/s13661-020-01433-1

31. Rezapour, S., Samei, M.E.: On the existence of solutions for a multi-singular pointwise defined fractional q-integro-differential equation. Bound. Value Probl. 2020, 38 (2020). https://doi.org/10.1186/s13661-020-01342-3

32. Goodrich, C., Peterson, A.C.: Discrete Fractional Calculus. Springer, Cham (2015). https://doi.org/10.1007/978-3-319-25562-0

33. Darzi, R., Agheli, B.: Existence results to positive solutions of fractional BVP with q-derivatives. J. Appl. Math. Comput. 55, 353-367 (2017) 
34. Samei, M.E., Hedayati, V., Rezapour, S.: Existence results for a fraction hybrid differential inclusion with Caputo-Hadamard type fractional derivative. Adv. Differ. Equ. 2019, 163 (2019). https://doi.org/10.1186/s13662-019-2090-8

35. Kac, V., Cheung, P.: Quantum Calculus. Universitext. Springer, New York (2002). https://doi.org/10.1007/978-1-4613-0071-7-1

36. Liang, S., Zhang, J.: Existence and uniqueness of positive solutions to $m$-point boundary value problem for nonlinear fractional differential equation. J. Appl. Math. Comput. 38(1), 225-241 (2012). https://doi.org/10.1186/s13661-020-01433-1

37. Bota, M.F., Karapinar, E., Mlesnite, O.: Ulam-Hyers stability results for fixed point problems via $\alpha$ - $\psi$-contractive mapping in b-metric space. Abstr. Appl. Anal. 2013, Article ID 825293 (2013). https://doi.org/10.1155/2013/825293

38. Karapinar, E., Panda, S.K., Lateef, D.: A new approach to the solution of Fredholm integral equation via fixed point on extended b-metric spaces. Symmetry 10(10), 512 (2018). https://doi.org/10.3390/sym10100512

39. Karapinar, E., Atangana, A., Fulga, A.: Pata type contractions involving rational expressions with an application to integral equations. Discrete Contin. Dyn. Syst. 14(10), 3629-3640 (2021). https://doi.org/10.3934/dcdss.2020420

40. Aksoy, U., Karapinar, E., Erhan, I.M.: Fixed point theorems in complete modular metric spaces and an application to anti-periodic boundary value problems. Filomat 31(17), 5475-5488 (2017). https://doi.org/10.2298/FIL1717475A

41. Aksoy, U., Karapinar, E., Erhan, I.M.: Fixed points of generalized $\alpha$-admissible contractions on $b$-metric spaces with an application to boundary value problems. J. Nonlinear Convex Anal. 17(6), 1095-1108 (2016)

42. Aydogan, S.M., Baleanu, D., Mousalou, A., Rezapour, S.: On high order fractional integro-differential equations including the Caputo-Fabrizio derivative. Bound. Value Probl. 2018, 90 (2018). https://doi.org/10.1186/s13661-018-1008-9

43. Baleanu, D., Mousalou, A., Rezapour, S.: On the existence of solutions for some infinite coefficient-symmetric Caputo-Fabrizio fractional integro-differential equations. Bound. Value Probl. 2017, 145 (2017). https://doi.org/10.1186/s13661-017-0867-9

44. Baleanu, D., Etemad, S., Rezapour, S.: On a fractional hybrid integro-differential equation with mixed hybrid integral boundary value conditions by using three operators. Alex. Eng. J. 59(5), 3019-3027 (2020). https://doi.org/10.1016/j.aej.2020.04.053

45. Mohammadi, H., Kumar, S., Rezapour, S., Etemad, S.: A theoretical study of the Caputo-Fabrizio fractional modeling for hearing loss due to Mumps virus with optimal control. Chaos Solitons Fractals 144, 110668 (2021). https://doi.org/10.1016/j.chaos.2021.110668

46. Samei, M.E., Yang, W.: Existence of solutions for $k$-dimensional system of multi-term fractional $q$-integro-differential equations under anti-periodic boundary conditions via quantum calculus. Math. Methods Appl. Sci. 43(7), 4360-4382 (2020). https://doi.org/10.1002/mma.6198

47. Samei, M.E.: Existence of solutions for a system of singular sum fractional $q$-differential equations via quantum calculus. Adv. Differ. Equ. 2019, 163 (2019). https://doi.org/10.1186/s13662-019-2480-y

48. Aydogan, M., Baleanu, D., Aguilar, J.F.G., Rezapour, S., Samei, M.E.: Approximate endpoint solutions for a class of fractional $q$-differential inclusions. Fractals 28(8), 2040029 (2020). https://doi.org/10.1142/S0218348X20400290

49. Ahmadi, A., Samei, M.E.: On existence and uniqueness of solutions for a class of coupled system of three term fractional q-differential equations. J. Adv. Math. Stud. 13(1), 69-80 (2020)

50. Su, X., Zhang, S.: Solution to boundary value problems for nonlinear differential equations of fractional order. Electron. J. Differ. Equ. 2009, 26 (2009)

51. Matar, M.M.: Boundary value problem for fractional integro-differential equations with nonlocal conditions. Int J. Open Probl. Comput. Sci. Math. 3(4), 481-489 (2010)

52. Tian, Y., Zhou, Y.: Positive solutions for multipoint boundary value problem of functional differential equations. J. Appl. Math. Comput. 38, 417-427 (2012)

53. Nyamoradi, N.: Multiple positive solutions for fractional differential systems. Ann. Univ. Ferrara 58(2), 359-369 (2012)

54. Sun, S., Li, Q., Li, Y.: Existence and uniqueness of solutions for a coupled system of multiterm nonlinear fractional differential equations. Comput. Math. Appl. 64(10), 3310-3320 (2012)

55. Houas, M., Benbachir, M.: Existence solutions for three point boundary value problem for differential equations. J. Fract. Calc. Appl. 6(1), 160-174 (2015)

56. Khan, R.A., Khan, H.: On existence of solution for multipoints boundary value problem. J. Fract. Calc. Appl. 5(2), 121-132 (2014)

57. Akrami, M.H., Erjaee, G.H.: Existence uniqueness and well-posed conditions on a class of fractional differentia equations with boundary condition. J. Fract. Calc. Appl. 6(2), 171-185 (2015). https://doi.org/10.3390/sym11050686

58. Diethelm, K.: The Analysis of Fractional Differential Equations. Springer, Berlin (2010)

59. Bohner, M., Peterson, A.: Dynamic Equations on Time Scales. Birkhäuser, Boston (2001)

60. Atici, F., Eloe, P.W.: Fractional q-calculus on a time scale. J. Nonlinear Math. Phys. 14(3), 341-352 (2007). https://doi.org/10.2991/jnmp.2007.14.3.4

61. Samei, M.E., Zanganeh, H., Aydogan, S.M.: Investigation of a class of the singular fractional integro-differential quantum equations with multi-step methods. J. Math. Ext. 17(1), 1-545 (2021)

62. Rajković, P.M., Marinković, S.D., Stanković, M.S.: Fractional integrals and derivatives in q-calculus. Appl. Anal. Discrete Math. 1, 311-323 (2007)

63. Podlubny, l.: Fractional Differential Equations. Academic Press, San Diego (1999)

64. Smart, D.R.: Fixed Point Theorems. Cambridge University Press, New York (1980) 